\title{
Stability of the Forward/Reverse\#Shock System Formed by the Impact of a Relativistic Fireball on an Ambient Medium
}

\section{Citation}

Wang, Xiaohu, Abraham Loeb, and Eli Waxman. 2002. "Stability of the Forward/Reverse\# Shock System Formed by the Impact of a Relativistic Fireball on an Ambient Medium." The Astrophysical Journal 568 (2): 830-44. https://doi.org/10.1086/339163.

\section{Permanent link}

http://nrs.harvard.edu/urn-3:HUL.InstRepos:41393326

\section{Terms of Use}

This article was downloaded from Harvard University's DASH repository, and is made available under the terms and conditions applicable to Other Posted Material, as set forth at http:// nrs.harvard.edu/urn-3:HUL.InstRepos:dash.current.terms-of-use\#LAA

\section{Share Your Story}

The Harvard community has made this article openly available. Please share how this access benefits you. Submit a story. 


\title{
Stability of the Forward/Reverse Shock System Formed by the Impact of a Relativistic Fireball on an Ambient Medium
}

\author{
Xiaohu Wang, Abraham Loeb \\ Astronomy Department, Harvard University, 60 Garden Street, Cambridge, MA 02138; \\ xwang,aloeb@cfa.harvard.edu \\ and Eli Waxman \\ Department of Condensed Matter Physics, Weizmann Institute, Rehovot 76100, Israel; \\ waxman@wicc.weizmann.ac.il
}

\begin{abstract}
We analyze the stability of a relativistic double (forward/reverse) shock system which forms when the fireball of a Gamma-Ray Burst (GRB) impacts on the surrounding medium. We find this shock system to be stable to linear global perturbations for either a uniform or a wind $\left(r^{-2}\right)$ density profile of the ambient medium. For the wind case, we calculate analytically the frequencies of the normal modes which could modulate the early short-term variability of GRB afterglows. We find that perturbations in the double shock system could induce oscillatory fluctuations in the observed flux on short (down to seconds) time scales during the early phase of an afterglow.
\end{abstract}

Subject headings: gamma rays: bursts — ISM

\section{Introduction}

Gamma-ray bursts (GRBs) and their afterglows are most naturally described by the relativistic "fireball" model (see e.g., Paczyński \& Rhoads 1993; Katz 1994; Mészáros \& Rees 1993, 1997; Waxman 1997a,b; Sari, Piran, \& Narayan 1998). In this model, a compact source releases a large amount of energy over a short time and produces a fireball which expands relativistically as a thin shell. When the shell encounters the circumburst medium, two shocks are formed - a forward shock which propagates into the circumburst medium and accelerates it, and a reverse shock which propagates into the relativistic shell and decelerates it (Mészáros \& Rees 1992; Katz 1994; Mészáros, Rees, \& Papathanassiou 1994; Sari \& Piran 1995; Sari, Narayan, \& Piran 1996). Later on, after a significant mass of circumburst medium is accumulated, the shell approaches a self-similar behaviour, as originally described by Blandford \& McKee (1976), where there is only one forward shock propagating into the circumburst medium. The circumburst medium could be either the interstellar medium (ISM) or a progenitor wind. 
The stability of the Blandford-McKee (1976) solution has been demonstrated recently by Gruzinov (2000). Here, we analyze the stability of a forward/reverse relativistic shock system. This double shock system exists during an important phase in the evolution of GRBs and its stability has observational consequences. In particular, oscillations or instabilities could translate to specific patterns of temporal variability in the lightcurves of GRB afterglows.

In our linear perturbation analysis we generalize the "thin shell" method first introduced by Vishniac (1983) in the non-relativistic regime. This method simplifies the equations describing the stability of a spherical shock when the wavelength of the perturbation is much larger than the thickness of the shocked shell. In our relativistic treatment we focus on global perturbations for which the wavelength is much larger than the thickness of the forward/reverse shock system. We consider the regime of GRB parameters where the reverse shock is relativistic (although in reality it may also be non-relativistic). In $\S 2$ we derive the perturbation equations for the forward/reverse shock system. In $\S 3$ we show the analytical results for the wind case and the numerical results for both the wind and ISM cases. Finally, in $\S 4$ we summarize our main conclusions.

\section{Linear Perturbation Equations}

The interaction between a relativistically expanding shell and the circumburst medium results in a double shock system, as shown in Figure 1. The system includes four distinct regions: the circumburst medium (region 1) and the shocked circumburst medium (region 2) are separated by the forward shock (shock 1), while the shocked material in the shell (region 3) and the unshocked material in the shell (region 4) are separated by the reverse shock (shock 2). Region 2 and 3 are separated by a contact discontinuity. Our analysis is done in the observer frame where the circumburst medium is at rest. We use a spherical coordinate system whose origin is located at the center of the explosion. The radii of the contact discontinuity, shock 1 and shock 2 are denoted by $R_{0}, R_{1}$ and $R_{2}$ respectively. We refer to the combination of region 2 and 3 as the layer whose stability we consider. Similarly to Vishniac (1983) we make the thin shell approximation, i.e. assume that

$$
\frac{R_{1}-R_{2}}{R_{0}}<k\left(R_{1}-R_{2}\right) \ll 1,
$$

where $k$ is the wavenumber of the perturbations. Note that although shock 1 is relativistic, shock 2 could be either relativistic or nonrelativistic. In this paper, we only consider the situation where shock 2 is relativistic.

The equations of motion for an ideal relativistic fluid are

$$
\begin{gathered}
\frac{\partial}{\partial t}(\gamma \rho)+\nabla \cdot(\gamma \rho \mathbf{u})=0 \\
\frac{\gamma^{2}}{c^{2}}(e+p)\left[\frac{\partial \mathbf{u}}{\partial t}+(\mathbf{u} \cdot \nabla) \mathbf{u}\right]+\left(\nabla p+\frac{\mathbf{u}}{c^{2}} \frac{\partial p}{\partial t}\right)=0
\end{gathered}
$$


where $\rho, \mathbf{u}, e, p$ and $\gamma$ are the fluid density, velocity, energy density, pressure and Lorentz factor, respectively. We define the surface density $\sigma$, bulk radial velocity $V_{r}$ and average tangential velocity $\mathbf{V}_{T}$ of region 2 as follows:

$$
\begin{gathered}
\sigma(\theta, \phi)=R_{0}^{-2} \int_{R_{0}}^{R_{1}} \gamma \rho r^{2} d r \\
V_{r}(\theta, \phi)=\left(\sigma R_{0}^{2}\right)^{-1} \int_{R_{0}}^{R_{1}} \gamma \rho u_{r} r^{2} d r \\
\mathbf{V}_{T}(\theta, \phi)=\left(\sigma R_{0}^{2}\right)^{-1} \int_{R_{0}}^{R_{1}} \gamma \rho \mathbf{u}_{T} r^{2} d r .
\end{gathered}
$$

The time evolution of these variables can be obtained by integrating equations (2) and (3) across region 2 , using the boundary conditions at shock 1 and at the contact discontinuity and neglecting terms of higher order in $\left(R_{1}-R_{2}\right) / R_{0}$ and $k\left(R_{1}-R_{2}\right)$. We get

$$
\begin{gathered}
\partial_{t} \sigma=-2 \frac{V_{r}}{R_{0}} \sigma+\rho_{1} c-\sigma \nabla_{T} \cdot \mathbf{V}_{T} \\
\partial_{t} \gamma\left(R_{0}\right)=-2 \sigma^{-1} \gamma\left(R_{0}\right) \rho_{1} c+\frac{3^{3 / 4}}{2^{1 / 2}} \sigma^{-1} \frac{p^{3 / 4}\left(R_{0}\right) \rho_{1}^{1 / 4}}{\gamma^{1 / 2}\left(R_{0}\right) c^{1 / 2}}+\frac{\gamma\left(R_{0}\right)}{2 c} V_{r} \nabla_{T} \cdot \mathbf{V}_{T}, \\
\partial_{t} \mathbf{V}_{T}=\frac{1}{3} \sigma^{-1} c^{2}\left[\rho_{1}-3^{3 / 4} \cdot 2^{1 / 2} \frac{p^{3 / 4}\left(R_{0}\right) \rho_{1}^{1 / 4}}{\gamma^{3 / 2}\left(R_{0}\right) c^{3 / 2}}\right] \nabla_{T} R_{0}-\sigma^{-1} \rho_{1} c \mathbf{V}_{T} \\
-\frac{V_{r}}{R_{0}} \mathbf{V}_{T}-\sigma^{-1} \frac{c^{2}}{3 \gamma^{2}\left(R_{0}\right)} \nabla_{T} \sigma+\frac{c^{2}}{3 \gamma^{3}\left(R_{0}\right)} \nabla_{T} \gamma\left(R_{0}\right),
\end{gathered}
$$

where $\rho_{1}$ is the density of the unshocked circumburst medium just in front of shock $1, \gamma\left(R_{0}\right)$ and $p\left(R_{0}\right)$ are the Lorentz factor and pressure at the contact discontinuity, and $\nabla_{T}$ denotes the tangential derivatives. In deriving the above equations we also assumed that the radial velocities are dominated by the bulk motion of regions 2 and 3 (denoted thereafter as the "shock layer"), so that $\dot{R}_{0} \approx V_{r}$. The full derivation of the above equations is given in the Appendix.

From equations (7) and (8) we obtain the unperturbed equations:

$$
\begin{gathered}
\partial_{t} \sigma^{(0)}=-2 \frac{v_{c}}{R_{0}^{(0)}} \sigma^{(0)}+\rho_{1} c \\
\partial_{t} \gamma_{c}=-2\left(\sigma^{(0)}\right)^{-1} \gamma_{c} \rho_{1} c+\frac{3^{3 / 4}}{2^{1 / 2}}\left(\sigma^{(0)}\right)^{-1} \frac{p_{c}^{3 / 4} \rho_{1}^{1 / 4}}{\gamma_{c}^{1 / 2} c^{1 / 2}},
\end{gathered}
$$

where $v_{c}=\dot{R}_{0}^{(0)}=V_{r}^{(0)}$ and $\gamma_{c}=\gamma^{(0)}\left(R_{0}\right)=1 / \sqrt{1-v_{c}^{2} / c^{2}}$ are the velocity and Lorentz factor of the unperturbed contact discontinuity, $p_{c}=p^{(0)}\left(R_{0}\right)$ is the unperturbed pressure at the contact discontinuity, and we use a superscript $(0)$ to denote unperturbed values. 
For the shocked shell in region 3, we define the surface density, bulk radial velocity and average tangential velocity to be:

$$
\begin{gathered}
\sigma_{3}(\theta, \phi)=R_{0}^{-2} \int_{R_{2}}^{R_{0}} \gamma \rho r^{2} d r \\
V_{r 3}(\theta, \phi)=\left(\sigma_{3} R_{0}^{2}\right)^{-1} \int_{R_{2}}^{R_{0}} \gamma \rho u_{r} r^{2} d r \\
\mathbf{V}_{T 3}(\theta, \phi)=\left(\sigma_{3} R_{0}^{2}\right)^{-1} \int_{R_{2}}^{R_{0}} \gamma \rho \mathbf{u}_{T} r^{2} d r
\end{gathered}
$$

where a subscript 3 denotes quantities in region 3 . The time derivatives of the above variables can be derived similarly to those in region 2 ,

$$
\begin{gathered}
\partial_{t} \sigma_{3}=-2 \frac{V_{r}}{R_{0}} \sigma_{3}+\frac{\gamma_{4}}{\gamma^{2}\left(R_{0}\right)} \rho_{4} c-\sigma_{3} \nabla_{T} \cdot \mathbf{V}_{T 3} \\
\partial_{t} \gamma\left(R_{0}\right)=\sigma_{3}^{-1} \frac{\gamma_{4}}{\gamma\left(R_{0}\right)} \rho_{4} c-3^{3 / 4} \sigma_{3}^{-1} \frac{p^{3 / 4}\left(R_{0}\right) \rho_{4}^{1 / 4} \gamma^{1 / 2}\left(R_{0}\right)}{\gamma_{4}^{1 / 2} c^{1 / 2}}+\frac{\gamma\left(R_{0}\right)}{2 c} V_{r} \nabla_{T} \cdot \mathbf{V}_{T 3} \\
\partial_{t} \mathbf{V}_{T 3}= \\
\quad \frac{1}{3} \sigma_{3}^{-1} c^{2}\left[2 \cdot 3^{3 / 4} \frac{p^{3 / 4}\left(R_{0}\right) \rho_{4}^{1 / 4}}{\gamma_{4}^{1 / 2} \gamma^{1 / 2}\left(R_{0}\right) c^{3 / 2}}-\frac{\gamma_{4}}{2 \gamma^{2}\left(R_{0}\right)} \rho_{4}\right] \nabla_{T} R_{0} \\
-\sigma_{3}^{-1} \frac{\gamma_{4}}{\gamma^{2}\left(R_{0}\right)} \rho_{4} c \mathbf{V}_{T 3}-\frac{V_{r}}{R_{0}} \mathbf{V}_{T 3}-\frac{1}{4 \gamma^{2}\left(R_{0}\right)}\left(\frac{\partial_{t} \rho_{4}}{\rho_{4}}\right) \mathbf{V}_{T 3} \\
-\sigma_{3}^{-1} \frac{c^{2}}{3 \gamma^{2}\left(R_{0}\right)} \nabla_{T} \sigma_{3}+\frac{c^{2}}{3 \gamma^{3}\left(R_{0}\right)} \nabla_{T} \gamma\left(R_{0}\right)
\end{gathered}
$$

where $\rho_{4}$ and $\gamma_{4}$ are the density and Lorentz factor of the unshocked shell (region 4) just in front of shock 2 . We have also used the relation $V_{r 3} \approx V_{r}$, as appropriate in the thin shell approximation.

The unperturbed equations for region 3 are

$$
\begin{gathered}
\partial_{t} \sigma_{3}^{(0)}=-2 \frac{v_{c}}{R_{0}^{(0)}} \sigma_{3}^{(0)}+\frac{\gamma_{4}}{\gamma_{c}^{2}} \rho_{4} c \\
\partial_{t} \gamma_{c}=\left(\sigma_{3}^{(0)}\right)^{-1} \frac{\gamma_{4}}{\gamma_{c}} \rho_{4} c-3^{3 / 4}\left(\sigma_{3}^{(0)}\right)^{-1} \frac{p_{c}^{3 / 4} \rho_{4}^{1 / 4} \gamma_{c}^{1 / 2}}{\gamma_{4}^{1 / 2} c^{1 / 2}}
\end{gathered}
$$

Equations (7), (8), (9), (15), (16) and (17) make a complete set of equations for the evolution of the forward/reverse shock system. The perturbation equations depend on the density profile of the circumburst medium. In the following subsections, we shall derive the perturbation equations for a uniform medium (such as the ISM) and for a progenitor wind. 


\subsection{Uniform Medium (ISM)}

For a uniform circumburst medium, $\rho_{1}=$ const. We define the perturbation variables $\delta \equiv \sigma / \sigma^{(0)}-1, \delta_{3} \equiv \sigma_{3} / \sigma_{3}^{(0)}-1, \Delta R \equiv R_{0}-R_{0}^{(0)}$ and $\Delta_{p} \equiv p\left(R_{0}\right) / p^{(0)}\left(R_{0}\right)-1$. From equations (77), (8) and (9), we obtain the following perturbation equations:

$$
\begin{gathered}
\partial_{t} \delta=-\frac{2}{R_{0}^{(0)}} \partial_{t} \Delta R+2 \frac{v_{c}}{\left(R_{0}^{(0)}\right)^{2}} \Delta R-\left(\sigma^{(0)}\right)^{-1} \rho_{1} c \delta-\nabla_{T} \cdot \mathbf{V}_{T}, \\
\partial_{t}^{2} \Delta R=\left[2\left(\sigma^{(0)}\right)^{-1} \frac{\rho_{1} c^{2}}{\gamma_{c}^{2}}-\frac{3^{3 / 4}}{2^{1 / 2}}\left(\sigma^{(0)}\right)^{-1} \frac{p_{c}^{3 / 4} \rho_{1}^{1 / 4} c^{1 / 2}}{\gamma_{c}^{7 / 2}}\right] \delta \\
+\left[4\left(\sigma^{(0)}\right)^{-1} \rho_{1} c-\frac{7 \cdot 3^{3 / 4}}{2^{3 / 2}}\left(\sigma^{(0)}\right)^{-1} \frac{p_{c}^{3 / 4} \rho_{1}^{1 / 4}}{\gamma_{c}^{3 / 2} c^{1 / 2}}\right] \partial_{t} \Delta R \\
+\frac{3^{7 / 4}}{2^{5 / 2}}\left(\sigma^{(0)}\right)^{-1} \frac{p_{c}^{3 / 4} \rho_{1}^{1 / 4} c^{1 / 2}}{\gamma_{c}^{7 / 2}} \Delta_{p}+\frac{v_{c}}{2 \gamma_{c}^{2}} \nabla_{T} \cdot \mathbf{V}_{T}, \\
\partial_{t} \mathbf{V}_{T}=\frac{1}{3}\left(\sigma^{(0)}\right)^{-1} c^{2}\left[\rho_{1}-3^{3 / 4} \cdot 2^{1 / 2} \frac{p_{c}^{3 / 4} \rho_{1}^{1 / 4}}{\gamma_{c}^{3 / 2} c^{3 / 2}}\right] \nabla_{T} \Delta R-\left(\sigma^{(0)}\right)^{-1} \rho_{1} c \mathbf{V}_{T} \\
-\frac{v_{c}}{R_{0}^{(0)}} \mathbf{V}_{T}-\frac{c^{2}}{3 \gamma_{c}^{2}} \nabla_{T} \delta+\frac{c}{3} \nabla_{T}\left(\partial_{t} \Delta R\right) .
\end{gathered}
$$

Assuming that $\gamma_{4}$ is a constant and there is no shell spreading, we get $\rho_{4} \propto R^{-2}$. Using this scaling we derive the following perturbation equations from equations (15), (16) and (17):

$$
\begin{aligned}
\partial_{t} \delta_{3}= & -2\left(\sigma_{3}^{(0)}\right)^{-1} \gamma_{4} \rho_{4} \partial_{t} \Delta R+\left[2 \frac{v_{c}}{\left(R_{0}^{(0)}\right)^{2}}-2\left(\sigma_{3}^{(0)}\right)^{-1} \frac{\gamma_{4}}{\gamma_{c}^{2}} \frac{\rho_{4} c}{R_{0}^{(0)}}\right] \Delta R \\
& -\left(\sigma_{3}^{(0)}\right)^{-1} \frac{\gamma_{4}}{\gamma_{c}^{2}} \rho_{4} c \delta_{3}-\nabla_{T} \cdot \mathbf{V}_{T 3}, \\
\partial_{t}^{2} \Delta R= & {\left[-\left(\sigma_{3}^{(0)}\right)^{-1} \frac{\gamma_{4}}{\gamma_{c}^{4}} \rho_{4} c^{2}+3^{3 / 4}\left(\sigma_{3}^{(0)}\right)^{-1} \frac{p_{c}^{3 / 4} \rho_{4}^{1 / 4} c^{1 / 2}}{\gamma_{4}^{1 / 2} \gamma_{c}^{5 / 2}}\right] \delta_{3} } \\
& +\left[-4\left(\sigma_{3}^{(0)}\right)^{-1} \frac{\gamma_{4}}{\gamma_{c}^{2}} \rho_{4} c+\frac{5 \cdot 3^{3 / 4}}{2}\left(\sigma_{3}^{(0)}\right)^{-1} \frac{p_{c}^{3 / 4} \rho_{4}^{1 / 4}}{\gamma_{4}^{1 / 2} \gamma_{c}^{1 / 2} c^{1 / 2}}\right] \partial_{t} \Delta R \\
& +\left[-2\left(\sigma_{3}^{(0)}\right)^{-1} \frac{\gamma_{4}}{\gamma_{c}^{4}} \frac{\rho_{4} c^{2}}{R_{0}^{(0)}}+\frac{3^{3 / 4}}{2}\left(\sigma_{3}^{(0)}\right)^{-1} \frac{p_{c}^{3 / 4} \rho_{4}^{1 / 4} c^{1 / 2}}{\gamma_{4}^{1 / 2} \gamma_{c}^{5 / 2}} \frac{1}{R_{0}^{(0)}}\right] \Delta R \\
& -\frac{3^{7 / 4}}{4}\left(\sigma_{3}^{(0)}\right)^{-1} \frac{p_{c}^{3 / 4} \rho_{4}^{1 / 4} c^{1 / 2}}{\gamma_{4}^{1 / 2} \gamma_{c}^{5 / 2}} \Delta_{p}+\frac{v_{c}}{2 \gamma_{c}^{2}} \nabla_{T} \cdot \mathbf{V}_{T 3},
\end{aligned}
$$




$$
\begin{aligned}
\partial_{t} \mathbf{V}_{T 3}= & \frac{1}{3}\left(\sigma_{3}^{(0)}\right)^{-1} c^{2}\left[2 \cdot 3^{3 / 4} \frac{p_{c}^{3 / 4} \rho_{4}^{1 / 4}}{\gamma_{4}^{1 / 2} \gamma_{c}^{1 / 2} c^{3 / 2}}-\frac{\gamma_{4}}{2 \gamma_{c}^{2}} \rho_{4}\right] \nabla_{T} \Delta R-\left(\sigma_{3}^{(0)}\right)^{-1} \frac{\gamma_{4}}{\gamma_{c}^{2}} \rho_{4} c \mathbf{V}_{T 3} \\
& -\frac{v_{c}}{R_{0}^{(0)}} \mathbf{V}_{T 3}-\frac{1}{4 \gamma_{c}^{2}}\left(\frac{\partial_{t} \rho_{4}}{\rho_{4}}\right) \mathbf{V}_{T 3}-\frac{c^{2}}{3 \gamma_{c}^{2}} \nabla_{T} \delta_{3}+\frac{c}{3} \nabla_{T}\left(\partial_{t} \Delta R\right) .
\end{aligned}
$$

In total, we have six perturbation equations (20)-(25) in six variables: $\delta, \delta_{3}, \Delta R, \mathbf{V}_{T}, \mathbf{V}_{T 3}$ and $\Delta_{p}$. In order to solve these equations, we first need to find the unperturbed values $\sigma^{(0)}, \sigma_{3}^{(0)}$, $\gamma_{c}$ and $p_{c}$ from equations (10), (11), (18) and (19). The time dependence of $\gamma_{c}$ has been derived by Sari \& Piran (1995) and Sari et al. (1996). When both the forward shock and the reverse shock are ultrarelativistic and strong,

$$
\gamma_{c} \propto \gamma_{4}^{1 / 2} f^{1 / 4}
$$

where $f=\rho_{4} / \rho_{1}$. For $\rho_{1}=$ const, $\gamma_{4}=$ const and $\rho_{4} \propto R^{-2}$, we get

$$
\gamma_{c} \propto R^{-1 / 2} \propto t^{-1 / 2}
$$

With $v_{c} \approx c$ and $R_{0}^{(0)} \approx c t$, equation (10) yields

$$
\sigma^{(0)} \approx \frac{1}{3} \rho_{1} c t .
$$

For $\rho_{4} \propto t^{-2}$ and $\gamma_{c} \propto t^{-1 / 2}$, equation (18) gives

$$
\sigma_{3}^{(0)} \approx \frac{1}{2} \frac{\gamma_{4}}{\gamma_{c}^{2}} \rho_{4} c t=\text { const } .
$$

By substituting equation (28) into equation (11) we find

$$
p_{c} \approx \frac{11^{4 / 3}}{3^{7 / 3} \cdot 2^{2 / 3}} \gamma_{c}^{2} \rho_{1} c^{2}=1.187 \gamma_{c}^{2} \rho_{1} c^{2}<\frac{4}{3} \gamma_{c}^{2} \rho_{1} c^{2},
$$

where $(4 / 3) \gamma_{c}^{2} \rho_{1} c^{2}$ is the pressure just behind the forward shock. Similarly, by substituting equation (29) into equation (19) we get

$$
p_{c} \approx \frac{5^{4 / 3}}{4^{4 / 3} \cdot 3} \frac{\gamma_{4}^{2}}{\gamma_{c}^{2}} \rho_{4} c^{2}=0.449 \frac{\gamma_{4}^{2}}{\gamma_{c}^{2}} \rho_{4} c^{2}>\frac{4}{3}{\overline{\gamma_{3}}}^{2} \rho_{4} c^{2},
$$

where $\overline{\gamma_{3}} \approx \gamma_{4} /\left(2 \gamma_{c}\right)$ is the Lorentz factor of the shocked shell (region 3) with respect to the unshocked shell (region 4) and $(4 / 3){\overline{\gamma_{3}}}^{2} \rho_{4} c^{2}$ is the pressure just behind the reverse shock. The pressure difference between the two sides of the layer causes it to decelerate. By combining equations (30) and (31) we find

$$
\gamma_{c} \approx 0.784 \gamma_{4}^{1 / 2}\left(\rho_{4} / \rho_{1}\right)^{1 / 4}
$$

Substitution of the values of $\sigma^{(0)}, \sigma_{3}^{(0)}$ and $p_{c}$ into the perturbation equations (20)-(25) yields,

$$
\partial_{t} \delta=-\frac{2}{c t} \partial_{t} \Delta R+\frac{2}{c t^{2}} \Delta R-\frac{3}{t} \delta-\nabla_{T} \cdot \mathbf{V}_{T},
$$




$$
\begin{gathered}
\partial_{t}^{2} \Delta R=\frac{1}{2} \frac{c}{\gamma_{c}^{2} t} \delta-\frac{29}{4} \frac{1}{t} \partial_{t} \Delta R+\frac{33}{8} \frac{c}{\gamma_{c}^{2} t} \Delta_{p}+\frac{c}{2 \gamma_{c}^{2}} \nabla_{T} \cdot \mathbf{V}_{T}, \\
\partial_{t} \mathbf{V}_{T}=-\frac{8}{3} \frac{c}{t} \nabla_{T} \Delta R-\frac{4}{t} \mathbf{V}_{T}-\frac{c^{2}}{3 \gamma_{c}^{2}} \nabla_{T} \delta+\frac{c}{3} \nabla_{T}\left(\partial_{t} \Delta R\right), \\
\partial_{t} \delta_{3}=-4 \frac{\gamma_{c}^{2}}{c t} \partial_{t} \Delta R-\frac{2}{c t^{2}} \Delta R-\frac{2}{t} \delta_{3}-\nabla_{T} \cdot \mathbf{V}_{T 3}, \\
\partial_{t}^{2} \Delta R=\frac{1}{2} \frac{c}{\gamma_{c}^{2} t} \delta_{3}-\frac{7}{4} \frac{1}{t} \partial_{t} \Delta R-\frac{11}{4} \frac{1}{\gamma_{c}^{2} t^{2}} \Delta R-\frac{15}{8} \frac{c}{\gamma_{c}^{2} t} \Delta_{p}+\frac{c}{2 \gamma_{c}^{2}} \nabla_{T} \cdot \mathbf{V}_{T 3}, \\
\partial_{t} \mathbf{V}_{T 3}=\frac{4}{3} \frac{c}{t} \nabla_{T} \Delta R-\frac{3}{t} \mathbf{V}_{T 3}-\frac{c^{2}}{3 \gamma_{c}^{2}} \nabla_{T} \delta_{3}+\frac{c}{3} \nabla_{T}\left(\partial_{t} \Delta R\right) .
\end{gathered}
$$

Combining equations (34) and (37) and eliminating $\Delta_{p}$, we get

$$
\begin{aligned}
\partial_{t}^{2} \Delta R= & \frac{5}{32} \frac{c}{\gamma_{c}^{2} t} \delta+\frac{11}{32} \frac{c}{\gamma_{c}^{2} t} \delta_{3}-\frac{111}{32} \frac{1}{t} \partial_{t} \Delta R-\frac{121}{64} \frac{1}{\gamma_{c}^{2} t^{2}} \Delta R \\
& +\frac{5}{32} \frac{c}{\gamma_{c}^{2}} \nabla_{T} \cdot \mathbf{V}_{T}+\frac{11}{32} \frac{c}{\gamma_{c}^{2}} \nabla_{T} \cdot \mathbf{V}_{T 3} .
\end{aligned}
$$

Next we expand the spatial dependence of the perturbation variables in spherical harmonics. We choose to normalize these variables so as to make them dimensionless and of a similar magnitude through the definitions

$$
\begin{gathered}
\Delta R=\sum_{l, m} \Delta R(l, m, t)\left[R_{0}^{(0)} / \gamma_{c}^{2}\right] Y_{l m}(\theta, \phi), \\
\delta=\sum_{l, m} \delta(l, m, t) Y_{l m}(\theta, \phi), \\
\delta_{3}=\sum_{l, m} \delta_{3}(l, m, t) Y_{l m}(\theta, \phi), \\
\mathbf{V}_{T}=\sum_{l, m} V_{T}(l, m, t)\left[c R_{0}^{(0)} / \gamma_{c}\right] \frac{\nabla_{T} Y_{l m}(\theta, \phi)}{l}, \\
\mathbf{V}_{T 3}=\sum_{l, m} V_{T 3}(l, m, t)\left[c R_{0}^{(0)} / \gamma_{c}\right] \frac{\nabla_{T} Y_{l m}(\theta, \phi)}{l} .
\end{gathered}
$$

Equations (33), (35), (36), (38) and (39) can be rewritten as

$$
\begin{gathered}
d_{t} \Delta R=F, \\
d_{t} \delta=-\frac{2}{\gamma_{c}^{2}} F-\frac{2}{\gamma_{c}^{2} t} \Delta R-\frac{3}{t} \delta+\frac{(l+1)}{\gamma_{c} t} V_{T}, \\
d_{t} V_{T}=\frac{l}{3 \gamma_{c}} F-\frac{2 l}{\gamma_{c} t} \Delta R-\frac{l}{3 \gamma_{c} t} \delta-\frac{9}{2 t} V_{T},
\end{gathered}
$$




$$
\begin{gathered}
d_{t} \delta_{3}=-4 F-\frac{8}{t} \Delta R-\frac{2}{t} \delta_{3}+\frac{(l+1)}{\gamma_{c} t} V_{T 3}, \\
d_{t} V_{T 3}=\frac{l}{3 \gamma_{c}} F+\frac{2 l}{\gamma_{c} t} \Delta R-\frac{l}{3 \gamma_{c} t} \delta_{3}-\frac{7}{2 t} V_{T 3}, \\
d_{t} F=-\frac{239}{32 t} F-\frac{143}{16 t^{2}} \Delta R+\frac{5}{32 t^{2}} \delta+\frac{11}{32 t^{2}} \delta_{3}-\frac{5}{32} \frac{(l+1)}{\gamma_{c} t^{2}} V_{T}-\frac{11}{32} \frac{(l+1)}{\gamma_{c} t^{2}} V_{T 3} .
\end{gathered}
$$

The perturbation variables in the above six equations are dimensionless and only functions of time. We have added the variable $F$ so that all the equations will have the form of first order differential equations.

\subsection{Wind Medium}

If the circumburst medium is a progenitor wind, $\rho_{1} \propto R^{-2}$. Accordingly, the perturbation equations (20) and (21) need to be changed to

$$
\begin{aligned}
\partial_{t} \delta=-\frac{2}{R_{0}^{(0)}} \partial_{t} \Delta R+ & {\left[2 \frac{v_{c}}{\left(R_{0}^{(0)}\right)^{2}}-2\left(\sigma^{(0)}\right)^{-1} \frac{\rho_{1} c}{R_{0}^{(0)}}\right] \Delta R-\left(\sigma^{(0)}\right)^{-1} \rho_{1} c \delta-\nabla_{T} \cdot \mathbf{V}_{T} } \\
\partial_{t}^{2} \Delta R= & {\left[2\left(\sigma^{(0)}\right)^{-1} \frac{\rho_{1} c^{2}}{\gamma_{c}^{2}}-\frac{3^{3 / 4}}{2^{1 / 2}}\left(\sigma^{(0)}\right)^{-1} \frac{p_{c}^{3 / 4} \rho_{1}^{1 / 4} c^{1 / 2}}{\gamma_{c}^{7 / 2}}\right] \delta } \\
& +\left[4\left(\sigma^{(0)}\right)^{-1} \rho_{1} c-\frac{7 \cdot 3^{3 / 4}}{2^{3 / 2}}\left(\sigma^{(0)}\right)^{-1} \frac{p_{c}^{3 / 4} \rho_{1}^{1 / 4}}{\gamma_{c}^{3 / 2} c^{1 / 2}}\right] \partial_{t} \Delta R \\
& +\left[4\left(\sigma^{(0)}\right)^{-1} \frac{\rho_{1} c^{2}}{\gamma_{c}^{2} R_{0}^{(0)}}-\frac{3^{3 / 4}}{2^{3 / 2}}\left(\sigma^{(0)}\right)^{-1} \frac{p_{c}^{3 / 4} \rho_{1}^{1 / 4} c^{1 / 2}}{\gamma_{c}^{7 / 2} R_{0}^{(0)}}\right] \Delta R \\
& +\frac{3^{7 / 4}}{2^{5 / 2}}\left(\sigma^{(0)}\right)^{-1} \frac{p_{c}^{3 / 4} \rho_{1}^{1 / 4} c^{1 / 2}}{\gamma_{c}^{7 / 2}} \Delta_{p}+\frac{v_{c}}{2 \gamma_{c}^{2}} \nabla_{T} \cdot \mathbf{V}_{T}
\end{aligned}
$$

Equations (22)-(25) remain the same as in the uniform medium case.

Since $\rho_{1} \propto R^{-2}$ and $\rho_{4} \propto R^{-2}$ in the wind case, equation (26) implies that $\gamma_{c}$ is constant over time. Equations (10), (11), (18) and (19) then yield the unperturbed parameters:

$$
\begin{gathered}
\sigma^{(0)} \approx \rho_{1} c t, \\
\sigma_{3}^{(0)} \approx \frac{\gamma_{4}}{\gamma_{c}^{2}} \rho_{4} c t, \\
p_{c} \approx \frac{4}{3} \gamma_{c}^{2} \rho_{1} c^{2} \approx \frac{1}{3} \frac{\gamma_{4}^{2}}{\gamma_{c}^{2}} \rho_{4} c^{2}, \\
\gamma_{c} \approx \frac{1}{\sqrt{2}} \gamma_{4}^{1 / 2}\left(\frac{\rho_{4}}{\rho_{1}}\right)^{1 / 4} .
\end{gathered}
$$


Substitution of equations (53)-(55) into the perturbation equations (51), (52) and (22)-(25), yields

$$
\begin{gathered}
\partial_{t} \delta=-\frac{2}{c t} \partial_{t} \Delta R-\frac{1}{t} \delta-\nabla_{T} \cdot \mathbf{V}_{T} \\
\partial_{t}^{2} \Delta R=-\frac{3}{t} \partial_{t} \Delta R+\frac{3}{\gamma_{c}^{2} t^{2}} \Delta R+\frac{3}{2} \frac{c}{\gamma_{c}^{2} t} \Delta_{p}+\frac{c}{2 \gamma_{c}^{2}} \nabla_{T} \cdot \mathbf{V}_{T}, \\
\partial_{t} \mathbf{V}_{T}=-\frac{c}{t} \nabla_{T} \Delta R-\frac{2}{t} \mathbf{V}_{T}-\frac{c^{2}}{3 \gamma_{c}^{2}} \nabla_{T} \delta+\frac{c}{3} \nabla_{T}\left(\partial_{t} \Delta R\right), \\
\partial_{t} \delta_{3}=-2 \frac{\gamma_{c}^{2}}{c t} \partial_{t} \Delta R-\frac{1}{t} \delta_{3}-\nabla_{T} \cdot \mathbf{V}_{T 3}, \\
\partial_{t}^{2} \Delta R=-\frac{3}{2} \frac{1}{t} \partial_{t} \Delta R-\frac{3}{2} \frac{1}{\gamma_{c}^{2} t^{2}} \Delta R-\frac{3}{4} \frac{c}{\gamma_{c}^{2} t} \Delta_{p}+\frac{c}{2 \gamma_{c}^{2}} \nabla_{T} \cdot \mathbf{V}_{T 3}, \\
\partial_{t} \mathbf{V}_{T 3}=\frac{1}{2} \frac{c}{t} \nabla_{T} \Delta R-\frac{2}{t} \mathbf{V}_{T 3}-\frac{c^{2}}{3 \gamma_{c}^{2}} \nabla_{T} \delta_{3}+\frac{c}{3} \nabla_{T}\left(\partial_{t} \Delta R\right) .
\end{gathered}
$$

Combining equations (58) and (61), and eliminating $\Delta_{p}$, we get

$$
\partial_{t}^{2} \Delta R=-\frac{2}{t} \partial_{t} \Delta R+\frac{c}{6 \gamma_{c}^{2}} \nabla_{T} \cdot \mathbf{V}_{T}+\frac{c}{3 \gamma_{c}^{2}} \nabla_{T} \cdot \mathbf{V}_{T 3}
$$

Using the same normalized perturbation variables as defined in equations (40)-(44), we can rewrite equations (57), (59), (60), (62) and (63) as

$$
\begin{gathered}
d_{t} \Delta R=F, \\
d_{t} \delta=-\frac{2}{\gamma_{c}^{2}} F-\frac{2}{\gamma_{c}^{2} t} \Delta R-\frac{1}{t} \delta+\frac{(l+1)}{\gamma_{c} t} V_{T}, \\
d_{t} V_{T}=\frac{l}{3 \gamma_{c}} F-\frac{2 l}{3 \gamma_{c} t} \Delta R-\frac{l}{3 \gamma_{c} t} \delta-\frac{2}{t} V_{T}, \\
d_{t} \delta_{3}=-2 F-\frac{2}{t} \Delta R-\frac{1}{t} \delta_{3}+\frac{(l+1)}{\gamma_{c} t} V_{T 3}, \\
d_{t} V_{T 3}=\frac{l}{3 \gamma_{c}} F+\frac{5 l}{6 \gamma_{c} t} \Delta R-\frac{l}{3 \gamma_{c} t} \delta_{3}-\frac{2}{t} V_{T 3}, \\
d_{t} F=-\frac{4}{t} F-\frac{2}{t^{2}} \Delta R-\frac{(l+1)}{6 \gamma_{c} t^{2}} V_{T}-\frac{(l+1)}{3 \gamma_{c} t^{2}} V_{T 3} .
\end{gathered}
$$

These six first order differential equations are the final perturbation equations for the wind case. 


\section{Solutions of the Perturbations Equations}

For the wind case, $\gamma_{c}=$ const, and we can solve the perturbation equations analytically. If we define $F=F^{\prime} / t$, then equations (64)-69) can be rewritten in a matrix form:

$$
d_{t}\left(\begin{array}{c}
F^{\prime} \\
\Delta R \\
\delta \\
V_{T} \\
\delta_{3} \\
V_{T 3}
\end{array}\right)=\frac{1}{t}\left(\begin{array}{cccccc}
-3 & -2 & 0 & -\frac{(l+1)}{6 \gamma_{c}} & 0 & -\frac{(l+1)}{3 \gamma_{c}} \\
1 & 0 & 0 & 0 & 0 & 0 \\
-\frac{2}{\gamma_{c}^{2}} & -\frac{2}{\gamma_{c}^{2}} & -1 & \frac{(l+1)}{\gamma_{c}} & 0 & 0 \\
\frac{l}{3 \gamma_{c}} & -\frac{2 l}{3 \gamma_{c}} & -\frac{l}{3 \gamma_{c}} & -2 & 0 & 0 \\
-2 & -2 & 0 & 0 & -1 & \frac{(l+1)}{\gamma_{c}} \\
\frac{l}{3 \gamma_{c}} & \frac{5 l}{6 \gamma_{c}} & 0 & 0 & -\frac{l}{3 \gamma_{c}} & -2
\end{array}\right)\left(\begin{array}{c}
F^{\prime} \\
\Delta R \\
\delta \\
V_{T} \\
\delta_{3} \\
V_{T 3}
\end{array}\right) .
$$

In matrix notation, the above equation is

$$
d_{t} \mathbf{y}=\frac{1}{t} \mathbf{A} \cdot \mathbf{y}
$$

where $\mathbf{y}$ is a vector, and $\mathbf{A}$ is a $6 \times 6$ time-independent matrix.

The matrix A can be diagonalized through the transformation

$$
\mathbf{X}^{-1} \cdot \mathbf{A} \cdot \mathbf{X}=\operatorname{diag}\left(\lambda_{1} \cdots \lambda_{6}\right)=\mathbf{D},
$$

where $\lambda_{1}, \cdots, \lambda_{6}$ are the eigenvalues of the matrix $\mathbf{A}$, and $\mathbf{X}$ is the matrix formed by columns from the eigenvectors (i.e. the $k$-th column of $\mathbf{X}$ is the eigenvector corresponding to the eigenvalue $\left.\lambda_{k}\right)$. Equation (71) can then be transformed to

$$
d_{t} \mathbf{y}=\frac{1}{t}\left(\mathbf{X} \cdot \mathbf{D} \cdot \mathbf{X}^{-1}\right) \cdot \mathbf{y} \Longrightarrow d_{t}\left(\mathbf{X}^{-1} \cdot \mathbf{y}\right)=\frac{1}{t} \mathbf{D} \cdot\left(\mathbf{X}^{-1} \cdot \mathbf{y}\right)
$$

By defining a new vector $\mathbf{y}^{\prime}=\mathbf{X}^{-1} \cdot \mathbf{y}$, we get the equation

$$
d_{t} \mathbf{y}^{\prime}=\frac{1}{t} \mathbf{D} \cdot \mathbf{y}^{\prime}
$$

which has six components

$$
d_{t} y_{k}^{\prime}=\frac{1}{t} \lambda_{k} y_{k}^{\prime}, \quad k=1, \cdots, 6 .
$$

Since $\lambda_{k}$ can be a complex number, we write $\lambda_{k}=a_{k}+i b_{k}$. The solution to equation (75) is then

$$
y_{k}^{\prime}=c_{k} t^{a_{k}+i b_{k}}=c_{k} t^{a_{k}} e^{i b_{k} \ln t},
$$

where $c_{k}$ is a constant dictated by the initial conditions. Each $y_{k}^{\prime}$ defines a mode of the shock system. There are six modes in total corresponding to six eigenvalues of the matrix $\mathbf{A}$. The real part of each eigenvalue dictates the overall temporal behavior of the mode, while the imaginary part determines its oscillation frequency. The vector $\mathbf{y}$ can be derived from the relation

$$
\mathbf{y}=\mathbf{X} \cdot \mathbf{y}^{\prime}
$$


Hence, each component of the vector $\mathbf{y}$ is a linear combination of the six different modes. The vector $\mathbf{c}$ whose components are $c_{k}$, can be obtained from the initial conditions

$$
\mathbf{c}=\mathbf{y}^{\prime}(t=1)=\mathbf{X}^{-1} \cdot \mathbf{y}(t=1)
$$

namely

$$
\left(\begin{array}{c}
c_{1} \\
c_{2} \\
c_{3} \\
c_{4} \\
c_{5} \\
c_{6}
\end{array}\right)=\mathbf{X}^{-1} \cdot\left(\begin{array}{c}
F^{\prime}(t=1) \\
\Delta R(t=1) \\
\delta(t=1) \\
V_{T}(t=1) \\
\delta_{3}(t=1) \\
V_{T 3}(t=1)
\end{array}\right) .
$$

The eigenvalues of the matrix $\mathbf{A}$ can be calculated for different values of $\gamma_{c}$ and $l$. Figure 2 shows the real and imaginary parts of the six eigenvalues as functions of $l$ for $\gamma_{c}=500$. Each row in the figure contains two panels, corresponding to the real and imaginary parts of a particular eigenvalue. The results show that for $l \leq 320$, all the six eigenvalues are real numbers, and so there are no oscillations. For $320<l \leq 432$, two eigenvalues are complex numbers and they are a pair of complex conjugates, implying that two modes are oscillating with the same frequency. For $l>432$, there are two pairs of complex conjugates. The transition from real eigenvalues to complex eigenvalues occurs when $l \sim \gamma_{c}$, as expected from the fact that oscillations are possible only when causality allows communication across the scale of a wavelength for modes with $l \gtrsim \gamma_{c}$.

For the thin shell approximation to be valid, we require that the wavelength of the perturbation be much larger than the thickness of the forward/reverse shock system in the shock frame. The thickness of the shock system is $\lesssim 2 R_{0} / \zeta \gamma_{c}^{2}$ in the observer frame, and thus $\lesssim 2 R_{0} / \zeta \gamma_{c}$ in the shock frame. Here $\zeta$ is a constant that ranges between $\sim 4$ and $\sim 12$ for the wind and ISM profiles, respectively. The wavelength of the perturbation is $\sim 2 \pi R_{0} / l$ in the shock frame. Therefore, we enforce an upper limit on $l$ of $\sim 10 \gamma_{c}$. Figure 2 shows that for all values of $l$, the real parts of the six eigenvalues are $\leq-1$. This implies that all modes are decaying faster or proportional to $t^{-1}$. Since each perturbation variable is a linear combination of the six modes, we conclude that all perturbation variables should also decay faster than or equal to $t^{-1}$. Thus the system is stable. Note that for large $\gamma_{c}$ we expect the results to depend only on $l / \gamma_{c}$ [see equation (70)], and so our particular choice of $\gamma_{c}=500$ can be scaled appropriately to other values of $\gamma_{c}$.

For $l \gg \gamma_{c}$ the eigenvalues admit the following analytical solutions,

$$
\begin{array}{ccc}
\lambda_{1}=-1, & \lambda_{2}=-\frac{3}{2}-\frac{1}{\sqrt{3}} \frac{l}{\gamma_{c}} i, & \lambda_{3}=-\frac{3}{2}+\frac{1}{\sqrt{3}} \frac{l}{\gamma_{c}} i, \\
\lambda_{4}=-\frac{19}{9}, & \lambda_{5}=-\frac{13}{9}+\frac{1}{\sqrt{2}} \frac{l}{\gamma_{c}} i, & \lambda_{6}=-\frac{13}{9}-\frac{1}{\sqrt{2}} \frac{l}{\gamma_{c}} i,
\end{array}
$$

while in the limit of $l \ll \gamma_{c}$,

$$
\lambda_{1}=-1, \quad \lambda_{2}=-2+\frac{1}{3} \frac{l^{2}}{\gamma_{c}^{2}}, \quad \lambda_{3}=-1-\frac{1}{3} \frac{l^{2}}{\gamma_{c}^{2}},
$$




$$
\lambda_{4}=-1-\frac{1}{2} \frac{l^{2}}{\gamma_{c}^{2}}, \quad \lambda_{5}=-2-\frac{1}{3 \sqrt{2}} \frac{l}{\gamma_{c}}, \quad \lambda_{6}=-2+\frac{1}{3 \sqrt{2}} \frac{l}{\gamma_{c}}
$$

We have calculated the corresponding eigenvectors numerically as shown in Figure 3 (for $\left.\gamma_{c}=500\right)$. Each row in the figure contains two panels which show the real and imaginary parts of one of the six components of the eigenvectors as functions of $l$. Different line types correspond to the six different eigenvectors. The complex eigenvectors are all scaled to have a unit magnitude. Since each eigenvector corresponds to a mode, the relative values of the six components of the eigenvector measure the physical significance of perturbations in different physical parameters for that mode. For example, the mode corresponding to the eigenvalue $\lambda_{1}=-1$ has the temporal behavior of $t^{-1}$; the eigenvector for this mode is $(-0.275,0.275,-0.824,0,0.412,0)$, implying that this mode does not involve $V_{T}$ and $V_{T 3}$ perturbations. Also note that this mode does not depend on $l$ while all other modes change with $l$.

Equations (64)-(69) can also be solved numerically. We normalize all initial values of the perturbation variables to unity. The temporal interval of the calculation is from $t=1$ to 30 , and $\gamma_{c}$ is chosen to be 500. The time $t=1$ marks the beginning of the double shock system. After the initial explosion, the fireball expands and accelerates to a relativistic speed. A cold shell is formed after the thermal energy of the fireball is converted to a bulk kinetic energy. The forward/reverse shock system is formed at radius $R_{t=1}$ where the circumburst medium starts to decelerate significantly the relativistic shell. This radius can be approximated as the radius at which the mass of the circumburst medium swept by the shell is comparable to $M_{0} / \eta$, where $M_{0}$ is the mass of the initial baryonic load of the fireball, and $\eta$ is the initial thermal Lorentz factor of the fireball. If the fireball has a total equivalent isotropic energy $E$, we have $M_{0}=E / \eta c^{2}$. Thus $t=1$ corresponds to a time $T_{t=1} \approx(1+z) R_{t=1} / 2 \gamma_{c}^{2} c$ after the GRB trigger in the observer frame, where $z$ is the cosmological redshift of the source. Figure 4 shows our results. The six panels show the time evolution of $\Delta R, d_{t} \Delta R, \delta, V_{T}, \delta_{3}$ and $V_{T 3}$. We show results for four different $l$ values, namely $l=5, l=50, l=500$ and $l=5 \times 10^{3}$. These plots indicate that all perturbation variables decay quickly with time. For small values of $l$ (e.g., $l=5$ and $l=50$ ) there are no oscillations. For large values of $l$ (e.g., $l=5 \times 10^{3}$ ) the oscillations exist but damp away quickly. These results are consistent with our analytical derivations. For $l=500$, the oscillations start to appear although they are not apparent in the plot because of their low frequency. For $l \gg \gamma_{c}$, we can calculate the frequencies of the oscillations using the eigenvalues listed in equations (80) and (81). The oscillations have the form $\exp \left(i \frac{1}{\sqrt{3}} \frac{l}{\gamma_{c}} \ln t\right)$ or $\exp \left(i \frac{1}{\sqrt{2}} \frac{l}{\gamma_{c}} \ln t\right)$, so that the oscillation period increases with time. For $l=10 \gamma_{c}$, the shortest period is $\sim 2.4$, corresponding to $2.4 T_{t=1}$ in the observer frame. Hence, the oscillations could produce fluctuations in the observed flux on time scales as short as a few times the starting time of the double shock system $T_{t=1}$.

In the ISM case, $\gamma_{c}$ is not constant and so we can not write equations (45)-(50) in a matrix form that admits an analytic solution. Instead, we had to solve these equations numerically. In order to test the validity of the perturbation equations and the numerical code, we compared the numerical results for a spherical perturbation with $l=0$ to the analytic solution derived 
by perturbing directly the radial equations of motion, and found an excellent agreement between the two calculations. Similarly to the wind case, we normalized all initial values of the perturbation variables to unity, and chose an initial $\gamma_{c}=500$. Our numerical results are shown in Figure 5 and resemble qualitatively the wind case. Overall, the perturbations decay rapidly with time and oscillations appear only for large values of $l$. Similarly to the wind case, for $l=10 \gamma_{c}$ the shortest period of the oscillations is $\sim 2$, corresponding to $\sim 2 T_{t=1}$ in the observer frame. If the equivalent isotropic energy of the fireball is $E=10^{52} \mathrm{ergs}$, the initial thermal Lorentz factor of the fireball is $\eta=10^{3}$, the number density of the ISM is $n=1 \mathrm{~cm}^{-3}$, then we have $R_{t=1}=\left(3 E / 4 \pi n m_{p} \eta^{2} c^{2}\right)^{1 / 3} \sim 1.2 \times 10^{16} \mathrm{~cm}$. This corresponds to $T_{t=1}=(1+z) R_{t=1} / 2 \gamma_{c}^{2} c \sim 0.8(1+z)$ sec. Thus, the time scales of the fluctuations in the observed flux could be as short as $\sim 2(1+z)$ sec.

\section{Discussion}

We have solved the perturbation equations describing the double (forward/reverse) shock system which forms during the impact of a highly relativistic fireball on a surrounding medium. For both a uniform and a wind $\left(1 / r^{2}\right)$ density profile of the ambient medium, we have found the shock system to be stable to global perturbations. We therefore do not expect the shock to fragment. Our results are limited to relativistic reverse shocks, and appear to differ qualitatively from previous results in the non-relativistic regime (Vishniac 1983).

Our results apply also to collimated outflows as long as the double shock system is formed at a time when the Lorentz factor of the outflow is larger than the collimation angle.

We derived the frequencies of the normal modes which could modulate the short-term variability at the early phase of GRB afterglows. The results imply that perturbations in the double shock system could produce fluctuations in the observed flux on time scales as short as a

few seconds for $\gamma_{c} \sim 500$ in the ISM case. These short term fluctuations could be supplemented by variability on much longer time scales due to density inhomogeneities in the ISM; such inhomogeneities can lead to variability on time scales of tens of minutes in the optical band and days in the radio (Wang \& Loeb 2000).

This work was supported in part by grants from the Israel-US BSF (BSF-9800343), NSF (AST-9900877; AST-0071019), and NASA (NAG5-7039; NAG5-7768).

\section{REFERENCES}

Blandford, R. D., \& McKee, C. F. 1976, Phys. Fluids, 19, 1130

Gruzinov, A. 2000, astro-ph/0012364 
Katz, J. I. 1994, ApJ, 422, 248

Kobayashi, S., Piran, T., \& Sari, R. 1999, ApJ, 513, 669

Mészáros, P., \& Rees, M. J. 1992, MNRAS, 258, 41P

. 1993, ApJ, 405, 278

Mészáros, P., Rees, M. J., \& Papathanassiou, H. 1994, ApJ, 432, 181

Paczyński, B., \& Rhoads, J. E. 1993, ApJ, 418, L5

Wang, X., \& Loeb, A. 2000, ApJ, 535, 788

Waxman, E. 1997a, ApJ, 485, L5

—. 1997b, ApJ, 489, L33

Sari, R., Narayan, R., \& Piran, T. 1996, ApJ, 473, 204

Sari, R., \& Piran, T. 1995, ApJ, 455, L143

Sari, R., Piran, T., \& Narayan, R. 1998, ApJ, 497, L17

Vishniac, E. T. 1983, ApJ, 274, 152 


\section{APPENDIX}

Here we provide full details for the derivation of equations (7)-(9) in $\S 2$. We start by listing the equations of motion for a relativistic fluid in spherical coordinates. The continuity equation reads

$$
\frac{\partial}{\partial t}(\gamma \rho)+\frac{1}{r^{2}} \frac{\partial}{\partial r}\left(r^{2} \gamma \rho u_{r}\right)+\frac{1}{r \sin \theta} \frac{\partial}{\partial \theta}\left(\sin \theta \gamma \rho u_{\theta}\right)+\frac{1}{r \sin \theta} \frac{\partial}{\partial \phi}\left(\gamma \rho u_{\phi}\right)=0,
$$

and the three components of the momentum equation are

$$
\begin{gathered}
\frac{\gamma^{2}}{c^{2}}(e+p)\left[\frac{\partial u_{r}}{\partial t}+u_{r} \frac{\partial u_{r}}{\partial r}+u_{\theta} \frac{1}{r} \frac{\partial u_{r}}{\partial \theta}+u_{\phi} \frac{1}{r \sin \theta} \frac{\partial u_{r}}{\partial \phi}-\frac{1}{r}\left(u_{\theta}^{2}+u_{\phi}^{2}\right)\right]+\frac{\partial p}{\partial r}+\frac{u_{r}}{c^{2}} \frac{\partial p}{\partial t}=0, \\
\frac{\gamma^{2}}{c^{2}}(e+p)\left[\frac{\partial u_{\theta}}{\partial t}+u_{r} \frac{\partial u_{\theta}}{\partial r}+u_{\theta} \frac{1}{r} \frac{\partial u_{\theta}}{\partial \theta}+u_{\phi} \frac{1}{r \sin \theta} \frac{\partial u_{\theta}}{\partial \phi}+\frac{1}{r}\left(u_{r} u_{\theta}+\cot \theta u_{\phi}^{2}\right)\right]+\frac{1}{r} \frac{\partial p}{\partial \theta}+\frac{u_{\theta}}{c^{2}} \frac{\partial p}{\partial t}=0, \\
\frac{\gamma^{2}}{c^{2}}(e+p)\left[\frac{\partial u_{\phi}}{\partial t}+u_{r} \frac{\partial u_{\phi}}{\partial r}+u_{\theta} \frac{1}{r} \frac{\partial u_{\phi}}{\partial \theta}+u_{\phi} \frac{1}{r \sin \theta} \frac{\partial u_{\phi}}{\partial \phi}+\frac{1}{r}\left(u_{r} u_{\phi}+\cot \theta u_{\theta} u_{\phi}\right)\right]+\frac{1}{r \sin \theta} \frac{\partial p}{\partial \phi}+\frac{u_{\phi}}{c^{2}} \frac{\partial p}{\partial t}=0,
\end{gathered}
$$

where $\rho, e, p$ and $\gamma$ are the fluid density, energy density, pressure and Lorentz factor respectively, $u_{r}, u_{\theta}$ and $u_{\phi}$ are the three components of the fluid velocity.

For the forward/reverse shock system under consideration (see Figure 1), we define the following shell-averaged variables for region 2 :

$$
\begin{gathered}
\sigma(\theta, \phi)=R_{0}^{-2} \int_{R_{0}}^{R_{1}} \gamma \rho r^{2} d r \\
V_{r}(\theta, \phi)=\left(\sigma R_{0}^{2}\right)^{-1} \int_{R_{0}}^{R_{1}} \gamma \rho u_{r} r^{2} d r \\
\mathbf{V}_{T}(\theta, \phi)=\left(\sigma R_{0}^{2}\right)^{-1} \int_{R_{0}}^{R_{1}} \gamma \rho \mathbf{u}_{T} r^{2} d r
\end{gathered}
$$

where $\mathbf{u}_{T}$ is the tangential velocity vector.

Since the shocked material is relativistic, we adopt the relativistic equation of state, $p=e / 3$, in region 2. Equation (11) yields the evolution of the surface density

$$
\begin{aligned}
\partial_{t} \sigma= & -2 \frac{\dot{R}_{0}}{R_{0}} \sigma+\left(\frac{R_{1}}{R_{0}}\right)^{2} \gamma\left(R_{1}\right) \rho\left(R_{1}\right) \dot{R}_{1}-\gamma\left(R_{0}\right) \rho\left(R_{0}\right) \dot{R}_{0}+R_{0}^{-2} \int_{R_{0}}^{R_{1}} \frac{\partial}{\partial t}(\gamma \rho) r^{2} d r \\
= & -2 \frac{\dot{R}_{0}}{R_{0}} \sigma+\left(\frac{R_{1}}{R_{0}}\right)^{2} \gamma\left(R_{1}\right) \rho\left(R_{1}\right)\left[\dot{R}_{1}-u_{r}\left(R_{1}\right)\right]+\gamma\left(R_{0}\right) \rho\left(R_{0}\right)\left[u_{r}\left(R_{0}\right)-\dot{R}_{0}\right] \\
& -R_{0}^{-2} \int_{R_{0}}^{R_{1}}\left[\nabla \cdot\left(\gamma \rho \mathbf{u}_{T}\right)\right] r^{2} d r .
\end{aligned}
$$

Since $r=R_{0}$ defines the contact discontinuity between the shocked shell and the shocked circumburst medium and there is no mass flow across the contact discontinuity, we get $u_{r}\left(R_{0}\right)=\dot{R}_{0}$. Because the forward shock is a strong relativistic shock, we have the following shock jump conditions at shock 1 :

$$
\gamma^{2}\left(R_{1}\right)=\gamma_{s 1}^{2} / 2
$$




$$
\rho\left(R_{1}\right) / \rho_{1}=4 \gamma\left(R_{1}\right)
$$

where $\gamma\left(R_{1}\right)$ and $\rho\left(R_{1}\right)$ are the Lorentz factor and density of the fluid just behind the shock front, $\gamma_{s 1}$ is the Lorentz factor of the shock front and $\rho_{1}$ is the density of the unshocked circumburst medium just in front of the shock front. In the highly relativistic regime,

$$
\begin{gathered}
\dot{R}_{1} \approx c\left(1-\frac{1}{2 \gamma_{s 1}^{2}}\right), \\
u_{r}\left(R_{1}\right) \approx c\left(1-\frac{1}{2 \gamma^{2}\left(R_{1}\right)}\right) .
\end{gathered}
$$

From equations (9)-(12), we obtain

$$
\gamma\left(R_{1}\right) \rho\left(R_{1}\right)\left[\dot{R}_{1}-u_{r}\left(R_{1}\right)\right] \approx \rho_{1} c .
$$

Thus, equation (8) can be rewritten as

$$
\partial_{t} \sigma=-2 \frac{\dot{R}_{0}}{R_{0}} \sigma+\left(\frac{R_{1}}{R_{0}}\right)^{2} \rho_{1} c-R_{0}^{-2} \int_{R_{0}}^{R_{1}}\left[\nabla \cdot\left(\gamma \rho \mathbf{u}_{T}\right)\right] r^{2} d r
$$

To linear order, the last integration term in the above equation can be approximated by

$$
-R_{0}^{-2} \int_{R_{0}}^{R_{1}}\left[\nabla \cdot\left(\gamma \rho \mathbf{u}_{T}\right)\right] r^{2} d r=-\sigma \nabla_{T} \cdot \mathbf{V}_{T}+R_{0}^{-2} \int_{R_{0}}^{R_{1}}\left[\nabla \cdot\left(\gamma \rho \mathbf{u}_{T}\right)\right]\left(\frac{r}{R_{0}}-1\right) r^{2} d r,
$$

where the operator $\nabla_{T} \equiv\left(\hat{\theta} / R_{0}\right)(\partial / \partial \theta)+\left(\hat{\phi} / R_{0}\right)(\partial / \partial \phi)$ acts as follows on a scalar $\Psi$ and a vector f:

$$
\begin{gathered}
\nabla_{T} \Psi=\frac{1}{R_{0}} \frac{\partial \Psi}{\partial \theta} \hat{\theta}+\frac{1}{R_{0} \sin \theta} \frac{\partial \Psi}{\partial \phi} \hat{\phi}, \\
\nabla_{T} \cdot \mathbf{f}=\frac{1}{R_{0} \sin \theta} \frac{\partial}{\partial \theta}\left(\sin \theta f_{\theta}\right)+\frac{1}{R_{0} \sin \theta} \frac{\partial f_{\phi}}{\partial \phi} .
\end{gathered}
$$

Note that the second term on the right-hand side of equation (15) is of higher order in $\left(R_{1}-R_{0}\right) / R_{0}$ than the preceding term, and hence can be ignored in the thin shell approximation. Thus equation (14) can be rewritten as

$$
\partial_{t} \sigma=-2 \frac{\dot{R}_{0}}{R_{0}} \sigma+\left(\frac{R_{1}}{R_{0}}\right)^{2} \rho_{1} c-\sigma \nabla_{T} \cdot \mathbf{V}_{T}
$$

Similarly to the above derivation, we obtain for the bulk radial velocity

$$
\begin{aligned}
\partial_{t} V_{r}(\theta, \phi)= & -\frac{\partial_{t} \sigma}{\sigma} V_{r}-2 \frac{\dot{R}_{0}}{R_{0}} V_{r}+\sigma^{-1}\left(\frac{R_{1}}{R_{0}}\right)^{2} \gamma\left(R_{1}\right) \rho\left(R_{1}\right) u_{r}\left(R_{1}\right)\left[\dot{R}_{1}-u_{r}\left(R_{1}\right)\right] \\
& +\sigma^{-1} \gamma\left(R_{0}\right) \rho\left(R_{0}\right) u_{r}\left(R_{0}\right)\left[u_{r}\left(R_{0}\right)-\dot{R}_{0}\right]-\left(\sigma R_{0}^{2}\right)^{-1} \int_{R_{0}}^{R_{1}}\left[\nabla \cdot\left(\gamma \rho \mathbf{u}_{T}\right)\right] u_{r} r^{2} d r \\
& -\left(\sigma R_{0}^{2}\right)^{-1} \int_{R_{0}}^{R_{1}} \frac{\rho c^{2}}{4 \gamma p}\left(\frac{\partial p}{\partial r}+\frac{u_{r}}{c^{2}} \frac{\partial p}{\partial t}\right) r^{2} d r-\left(\sigma R_{0}^{2}\right)^{-1} \int_{R_{0}}^{R_{1}} \gamma \rho\left(\mathbf{u}_{T} \cdot \nabla u_{r}\right) r^{2} d r \\
& +\left(\sigma R_{0}^{2}\right)^{-1} \int_{R_{0}}^{R_{1}} \gamma \rho \mathbf{u}_{T}^{2} r d r .
\end{aligned}
$$


The last two terms on the right-hand-side of the above equation are nonlinear. By substituting equations (13) and (14) into the above equation, and keeping terms to the linear order we get

$$
\begin{aligned}
\partial_{t} V_{r}(\theta, \phi)= & \sigma^{-1}\left(\frac{R_{1}}{R_{0}}\right)^{2} \rho_{1} c\left[u_{r}\left(R_{1}\right)-V_{r}\right] \\
& +\left[\left(\sigma R_{0}^{2}\right)^{-1} V_{r} \int_{R_{0}}^{R_{1}}\left[\nabla \cdot\left(\gamma \rho \mathbf{u}_{T}\right)\right] r^{2} d r-\left(\sigma R_{0}^{2}\right)^{-1} \int_{R_{0}}^{R_{1}} u_{r}\left[\nabla \cdot\left(\gamma \rho \mathbf{u}_{T}\right)\right] r^{2} d r\right] \\
& -\left(\sigma R_{0}^{2}\right)^{-1} \int_{R_{0}}^{R_{1}} \frac{\rho c^{2}}{4 \gamma p}\left(\frac{\partial p}{\partial r}+\frac{u_{r}}{c^{2}} \frac{\partial p}{\partial t}\right) r^{2} d r .
\end{aligned}
$$

In order to evaluate the integral in the last term of the above equation, we need the relation between $p$ and $\rho$ inside region 2. Since entropy is conserved in this region,

$$
\frac{d}{d t}\left(\frac{p}{\rho^{4 / 3}}\right)=0
$$

implying that $p / \rho^{4 / 3}$ remains constant for a given fluid particle. Hence, a fluid layer which is at a distance $x(t)$ from the contact discontinuity $\left(r=R_{0}\right)$ inside region 2 , maintains a constant $p / \rho^{4 / 3}$ over time and its value is decided by the Lorentz factor of shock 1 at the time when this layer first crossed shock 1. However, at a particular time, different layers across region 2 have different values of $p / \rho^{4 / 3}$. Assuming that region 2 is decelerating with $\gamma \propto r^{-1 / 2}$ (for the uniform ISM case), we get

$$
\frac{p(x)}{\rho^{4 / 3}(x)}=\frac{c^{2}}{3 \cdot 4^{1 / 3} \rho_{1}^{1 / 3}}\left[\frac{\gamma_{a} R_{a}^{1 / 2}}{\left(8 \gamma_{a}^{2} R_{a} x+R_{a}^{2}\right)^{1 / 4}}\right]^{2 / 3},
$$

where $\gamma_{a}$ and $R_{a}$ are the Lorentz factor and radius of region 2 at the initial time. Apparently, the dependence of $p(x) / \rho^{4 / 3}(x)$ on $x$ is very weak, and so within the context of the thin shell approximation we simply assume that $p / \rho^{4 / 3}$ is constant across region 2 at any given time. This assumption is indeed satisfied in the numerical simulations performed by Kobayashi, Piran, \& Sari (1999). In equation (22), the term $8 \gamma_{a}^{2} R_{a} x$ can be at most comparable to $R_{a}^{2}$ (this happens in the very last stage of the evolution when the reverse shock crosses the shell), and so the error introduced by our approximation is small. For the wind case, $\gamma \approx$ const, and we can also treat $p / \rho^{4 / 3}$ as a constant across region 2 .

We may now calculate the integral

$$
\begin{aligned}
\int_{R_{0}}^{R_{1}} \frac{\rho c^{2}}{4 \gamma p} \frac{\partial p}{\partial r} r^{2} d r & =\int_{R_{0}}^{R_{1}} \frac{c^{2}}{3 \gamma} \frac{\partial \rho}{\partial r} r^{2} d r=\frac{c^{2}}{3 \gamma\left(R_{0}\right)} \int_{R_{0}}^{R_{1}} \frac{\partial \rho}{\partial r} r^{2} d r \\
& \approx \frac{c^{2}}{3 \gamma\left(R_{0}\right)}\left[\rho\left(R_{1}\right) R_{1}^{2}-\rho\left(R_{0}\right) R_{0}^{2}-\int_{R_{0}}^{R_{1}} 2 r \rho d r\right]
\end{aligned}
$$

In the thin shell approximation, all radial velocities are dominated by the overall radial motion of the shock layer. Hence, $\gamma$ was treated as a constant and was taken out of the integral. For the uniform ISM case, the density difference between the two edges of region 2 is not small; hence, 
the last term inside the square brackets is of order $\left(R_{1}-R_{0}\right) / R_{0}$ times the difference between the previous two terms and so it can be neglected. For the wind case, there is no pressure gradient across region 2, and so the above integral vanishes. However, even in this case we can ignore the last term and keep only the first two terms, because later on we will replace $R_{1}$ with $R_{0}$, and so the difference between the first two terms will vanish.

Another relevant integral is

$$
\begin{aligned}
\int_{R_{0}}^{R_{1}} \frac{\rho c^{2}}{4 \gamma p} \frac{u_{r}}{c^{2}} \frac{\partial p}{\partial t} r^{2} d r= & \int_{R_{0}}^{R_{1}} \frac{c^{2}}{3 \gamma} \frac{u_{r}}{c^{2}} \frac{\partial \rho}{\partial t} r^{2} d r=\frac{V_{r}}{3 \gamma\left(R_{0}\right)} \int_{R_{0}}^{R_{1}} \frac{\partial \rho}{\partial t} r^{2} d r \\
= & \frac{V_{r}}{3 \gamma\left(R_{0}\right)}\left[\frac{\partial}{\partial t} \int_{R_{0}}^{R_{1}} \rho r^{2} d r-\dot{R}_{1} \rho\left(R_{1}\right) R_{1}^{2}+\dot{R}_{0} \rho\left(R_{0}\right) R_{0}^{2}\right] \\
= & \frac{V_{r}}{3 \gamma\left(R_{0}\right)}\left[\frac{\sigma}{\gamma\left(R_{0}\right)} 2 R_{0} \dot{R}_{0}+\frac{R_{0}^{2}}{\gamma\left(R_{0}\right)} \partial_{t} \sigma-\frac{R_{0}^{2} \sigma}{\gamma^{2}\left(R_{0}\right)} \partial_{t} \gamma\left(R_{0}\right)\right. \\
& \left.-\dot{R}_{1} \rho\left(R_{1}\right) R_{1}^{2}+\dot{R}_{0} \rho\left(R_{0}\right) R_{0}^{2}\right]
\end{aligned}
$$

In the above derivation we pulled $u_{r}$ out of the integration assuming that it equals $V_{r}$, as appropriate in the thin shell approximation. By substituting equation (18) into equation (24) and making use of the following two relations

$$
\begin{aligned}
& c^{2}-V_{r} \dot{R}_{0} \approx \frac{c^{2}}{\gamma^{2}\left(R_{0}\right)}, \\
& c^{2}-V_{r} \dot{R}_{1} \approx \frac{3 c^{2}}{4 \gamma^{2}\left(R_{0}\right)},
\end{aligned}
$$

we get

$$
\begin{aligned}
\int_{R_{0}}^{R_{1}} \frac{\rho c^{2}}{4 \gamma p}\left(\frac{\partial p}{\partial r}+\frac{u_{r}}{c^{2}} \frac{\partial p}{\partial t}\right) r^{2} d r= & \frac{4 R_{0}^{2}}{3 \gamma^{2}\left(R_{0}\right)} \rho_{1} c^{2}-\frac{R_{0}^{2}}{3 \gamma^{3}\left(R_{0}\right)} \rho\left(R_{0}\right) c^{2} \\
& -\sigma \frac{R_{0}^{2}}{3 \gamma^{2}\left(R_{0}\right)} V_{r} \nabla_{T} \cdot \mathbf{V}_{T}-\sigma \frac{R_{0}^{2}}{3 \gamma^{3}\left(R_{0}\right)} V_{r} \partial_{t} \gamma\left(R_{0}\right) .
\end{aligned}
$$

Thus, equation (20) is now changed to

$$
\begin{aligned}
\partial_{t} V_{r}= & \sigma^{-1}\left(\frac{R_{1}}{R_{0}}\right)^{2} \rho_{1} c\left[u_{r}\left(R_{1}\right)-V_{r}\right] \\
& +\left[\left(\sigma R_{0}^{2}\right)^{-1} V_{r} \int_{R_{0}}^{R_{1}}\left[\nabla \cdot\left(\gamma \rho \mathbf{u}_{T}\right)\right] r^{2} d r-\left(\sigma R_{0}^{2}\right)^{-1} \int_{R_{0}}^{R_{1}} u_{r}\left[\nabla \cdot\left(\gamma \rho \mathbf{u}_{T}\right)\right] r^{2} d r\right] \\
& -\sigma^{-1} \frac{4}{3 \gamma^{2}\left(R_{0}\right)} \rho_{1} c^{2}+\sigma^{-1} \frac{1}{3 \gamma^{3}\left(R_{0}\right)} \rho\left(R_{0}\right) c^{2}+\frac{1}{3 \gamma^{2}\left(R_{0}\right)} V_{r} \nabla_{T} \cdot \mathbf{V}_{T} \\
& +\frac{1}{3 \gamma^{3}\left(R_{0}\right)} V_{r} \partial_{t} \gamma\left(R_{0}\right) .
\end{aligned}
$$

In order to close the final equations, we can only have one free variable for the radial velocities. We use the approximation that $u_{r}$ is constant across region 2 with $V_{r}=u_{r}\left(R_{1}\right)$, as appropriate 
under the thin shell approximation. Hence, the first two terms in equation (28) both vanish, and we end up with the following equation,

$$
\partial_{t} V_{r}=-\sigma^{-1} \frac{4}{3 \gamma^{2}\left(R_{0}\right)} \rho_{1} c^{2}+\sigma^{-1} \frac{1}{3 \gamma^{3}\left(R_{0}\right)} \rho\left(R_{0}\right) c^{2}+\frac{1}{3 \gamma^{2}\left(R_{0}\right)} V_{r} \nabla_{T} \cdot \mathbf{V}_{T}+\frac{1}{3 \gamma^{3}\left(R_{0}\right)} V_{r} \partial_{t} \gamma\left(R_{0}\right)
$$

Since

$$
\frac{\partial_{t} \gamma\left(R_{0}\right)}{\gamma^{3}\left(R_{0}\right)}=\frac{V_{r}}{c^{2}} \partial_{t} V_{r}
$$

equation (29) can be rewritten as

$$
\partial_{t} \gamma\left(R_{0}\right)=-2 \sigma^{-1} \gamma\left(R_{0}\right) \rho_{1} c+\frac{1}{2} \sigma^{-1} \rho\left(R_{0}\right) c+\frac{\gamma\left(R_{0}\right)}{2 c} V_{r} \nabla_{T} \cdot \mathbf{V}_{T}
$$

Because $p / \rho^{4 / 3}$ is a constant across region 2 , we obtain the following relation

$$
\rho\left(R_{0}\right)=3^{3 / 4} \cdot 2^{1 / 2} \frac{p^{3 / 4}\left(R_{0}\right) \rho_{1}^{1 / 4}}{\gamma^{1 / 2}\left(R_{0}\right) c^{3 / 2}} .
$$

Using this result, we can rewrite equation (31) as

$$
\partial_{t} \gamma\left(R_{0}\right)=-2 \sigma^{-1} \gamma\left(R_{0}\right) \rho_{1} c+\frac{3^{3 / 4}}{2^{1 / 2}} \sigma^{-1} \frac{p^{3 / 4}\left(R_{0}\right) \rho_{1}^{1 / 4}}{\gamma^{1 / 2}\left(R_{0}\right) c^{1 / 2}}+\frac{\gamma\left(R_{0}\right)}{2 c} V_{r} \nabla_{T} \cdot \mathbf{V}_{T}
$$

For the tangential velocity we have

$$
\begin{aligned}
\partial_{t} \mathbf{V}_{T}(\theta, \phi)= & -\frac{\partial_{t} \sigma}{\sigma} \mathbf{V}_{T}-2 \frac{\dot{R}_{0}}{R_{0}} \mathbf{V}_{T}+\sigma^{-1}\left(\frac{R_{1}}{R_{0}}\right)^{2} \gamma\left(R_{1}\right) \rho\left(R_{1}\right) \mathbf{u}_{T}\left(R_{1}\right)\left[\dot{R}_{1}-u_{r}\left(R_{1}\right)\right] \\
& +\sigma^{-1} \gamma\left(R_{0}\right) \rho\left(R_{0}\right) \mathbf{u}_{T}\left(R_{0}\right)\left[u_{r}\left(R_{0}\right)-\dot{R}_{0}\right]-\left(\sigma R_{0}^{2}\right)^{-1} \int_{R_{0}}^{R_{1}} \gamma \rho \frac{u_{r} \mathbf{u}_{T}}{r} r^{2} d r \\
& -\left(\sigma R_{0}^{2}\right)^{-1} \int_{R_{0}}^{R_{1}} \frac{\rho c^{2}}{4 \gamma p}\left(\nabla_{T} p+\frac{\mathbf{u}_{T}}{c^{2}} \frac{\partial p}{\partial t}\right) r^{2} d r \\
& -\left(\sigma R_{0}^{2}\right)^{-1} \int_{R_{0}}^{R_{1}} \gamma \rho\left(\mathbf{u}_{T} \cdot \nabla \mathbf{u}_{T}\right) r^{2} d r-\left(\sigma R_{0}^{2}\right)^{-1} \int_{R_{0}}^{R_{1}} \mathbf{u}_{T} \nabla \cdot\left(\gamma \rho \mathbf{u}_{T}\right) r^{2} d r .
\end{aligned}
$$

Apparently the last two terms in the above equation are nonlinear and can be ignored. By substituting equations (13) and (18) into the above equation, we get

$$
\begin{aligned}
\partial_{t} \mathbf{V}_{T}= & \sigma^{-1}\left(\frac{R_{1}}{R_{0}}\right)^{2} \rho_{1} c\left[\mathbf{u}_{T}\left(R_{1}\right)-\mathbf{V}_{T}\right]+\mathbf{V}_{T} \nabla_{T} \cdot \mathbf{V}_{T}-\left(\sigma R_{0}^{2}\right)^{-1} \int_{R_{0}}^{R_{1}} \gamma \rho \frac{u_{r} \mathbf{u}_{T}}{r} r^{2} d r \\
& -\left(\sigma R_{0}^{2}\right)^{-1} \int_{R_{0}}^{R_{1}} \frac{\rho c^{2}}{4 \gamma p}\left(\nabla_{T} p+\frac{\mathbf{u}_{T}}{c^{2}} \frac{\partial p}{\partial t}\right) r^{2} d r
\end{aligned}
$$

The second term on the right-hand-side of equation (35) is nonlinear and can be neglected. In the thin shell approximation, the third term on the right-hand side of equation (35) can be 
approximated as $-V_{r} \mathbf{V}_{T} / R_{0}$. Using the shock jump conditions at shock 1 and making use of the fact that the tangential velocities must be continuous across the shock front, we obtain

$$
\mathbf{u}_{T}\left(R_{1}\right)=-u_{r}\left(R_{1}\right)\left(\nabla_{T} R_{1}\right)
$$

Based on these considerations, equation (35) can be rewritten as

$$
\begin{aligned}
\partial_{t} \mathbf{V}_{T}= & \sigma^{-1}\left(\frac{R_{1}}{R_{0}}\right)^{2} \rho_{1} c\left[-u_{r}\left(R_{1}\right)\left(\nabla_{T} R_{1}\right)-\mathbf{V}_{T}\right]-\frac{V_{r}}{R_{0}} \mathbf{V}_{T} \\
& -\left(\sigma R_{0}^{2}\right)^{-1} \int_{R_{0}}^{R_{1}} \frac{\rho c^{2}}{4 \gamma p}\left(\nabla_{T} p+\frac{\mathbf{u}_{T}}{c^{2}} \frac{\partial p}{\partial t}\right) r^{2} d r
\end{aligned}
$$

Next we consider the integration term in the above equation which includes

$$
\begin{aligned}
\int_{R_{0}}^{R_{1}} \frac{\rho c^{2}}{4 \gamma p}\left(\nabla_{T} p\right) r^{2} d r= & \int_{R_{0}}^{R_{1}} \frac{c^{2}}{3 \gamma}\left(\nabla_{T} \rho\right) r^{2} d r \\
= & \int_{R_{0}}^{R_{1}} \frac{c^{2}}{3 \gamma^{2}}\left[\nabla_{T}(\gamma \rho)\right] r^{2} d r-\int_{R_{0}}^{R_{1}} \frac{\rho c^{2}}{3 \gamma^{2}}\left(\nabla_{T} \gamma\right) r^{2} d r \\
= & \frac{c^{2}}{3 \gamma^{2}\left(R_{0}\right)}\left[\nabla_{T} \int_{R_{0}}^{R_{1}} \gamma \rho r^{2} d r-\gamma\left(R_{1}\right) \rho\left(R_{1}\right) R_{1}^{2}\left(\nabla_{T} R_{1}\right)\right. \\
& \left.+\gamma\left(R_{0}\right) \rho\left(R_{0}\right) R_{0}^{2}\left(\nabla_{T} R_{0}\right)\right]-\frac{c^{2} \nabla_{T} \gamma\left(R_{0}\right)}{3 \gamma^{3}\left(R_{0}\right)} \int_{R_{0}}^{R_{1}} \gamma \rho r^{2} d r \\
= & \frac{c^{2}}{3 \gamma^{2}\left(R_{0}\right)}\left[R_{0}^{2} \nabla_{T} \sigma+2 \sigma R_{0}\left(\nabla_{T} R_{0}\right)-\gamma\left(R_{1}\right) \rho\left(R_{1}\right) R_{1}^{2}\left(\nabla_{T} R_{1}\right)\right. \\
& \left.+\gamma\left(R_{0}\right) \rho\left(R_{0}\right) R_{0}^{2}\left(\nabla_{T} R_{0}\right)\right]-\frac{c^{2}}{3 \gamma^{3}\left(R_{0}\right)} R_{0}^{2} \sigma \nabla_{T} \gamma\left(R_{0}\right) .
\end{aligned}
$$

In the last pair of square brackets of equation (38), the second term is much smaller than the fourth term and so it can be neglected. Another integration term is

$$
\begin{aligned}
\int_{R_{0}}^{R_{1}} \frac{\rho c^{2}}{4 \gamma p} \frac{\mathbf{u}_{T}}{c^{2}} \frac{\partial p}{\partial t} r^{2} d r & =\int_{R_{0}}^{R_{1}} \frac{1}{4 \gamma^{2}}\left(\partial_{t} \ln p\right) \gamma \rho \mathbf{u}_{T} r^{2} d r \\
& \approx \frac{1}{4 \gamma^{2}\left(R_{0}\right)}\left[\partial_{t} \ln p\left(R_{1}\right)\right] \int_{R_{0}}^{R_{1}} \gamma \rho \mathbf{u}_{T} r^{2} d r \\
& =\frac{\partial_{t} \gamma\left(R_{0}\right)}{2 \gamma^{3}\left(R_{0}\right)} \sigma R_{0}^{2} \mathbf{V}_{T}+\frac{1}{4 \gamma^{2}\left(R_{0}\right)}\left(\frac{\partial_{t} \rho_{1}}{\rho_{1}}\right) \sigma R_{0}^{2} \mathbf{V}_{T}
\end{aligned}
$$

Because $\partial_{t} \ln p$ does not change much across region 2, we took it out of the integration in the above derivation.

By substituting equation (31) into equation (39), we get

$$
\int_{R_{0}}^{R_{1}} \frac{\rho c^{2}}{4 \gamma p} \frac{\mathbf{u}_{T}}{c^{2}} \frac{\partial p}{\partial t} r^{2} d r \approx-\frac{R_{0}^{2}}{\gamma^{2}\left(R_{0}\right)} \rho_{1} c \mathbf{V}_{T}+\frac{R_{0}^{2}}{4 \gamma^{3}\left(R_{0}\right)} \rho\left(R_{0}\right) c \mathbf{V}_{T}+\frac{1}{4 \gamma^{2}\left(R_{0}\right)}\left(\frac{\partial_{t} \rho_{1}}{\rho_{1}}\right) \sigma R_{0}^{2} \mathbf{V}_{T}
$$


Now, by substituting equations (38) and (40) into equation (37), we get

$$
\begin{aligned}
\partial_{t} \mathbf{V}_{T}= & \frac{1}{3} \sigma^{-1} c^{2}\left[\rho_{1}-\frac{\rho\left(R_{0}\right)}{\gamma\left(R_{0}\right)}\right] \nabla_{T} R_{0}-\sigma^{-1} \rho_{1} c \mathbf{V}_{T}-\frac{V_{r}}{R_{0}} \mathbf{V}_{T} \\
& -\sigma^{-1} \frac{c^{2}}{3 \gamma^{2}\left(R_{0}\right)} \nabla_{T} \sigma+\frac{c^{2}}{3 \gamma^{3}\left(R_{0}\right)} \nabla_{T} \gamma\left(R_{0}\right)-\frac{1}{4 \gamma^{2}\left(R_{0}\right)}\left(\frac{\partial_{t} \rho_{1}}{\rho_{1}}\right) \mathbf{V}_{T}
\end{aligned}
$$

In deriving the above equation, we made the assumption that surface irregularities due to variations in the thickness of the shock layer is of higher order than irregularities due to the bulk displacement of the shock layer (regions $2 \& 3$ in Figure 1), so $\nabla_{T} R_{0}=\nabla_{T} R_{1}=\nabla_{T} R_{2}$. This is appropriate under the thin shell approximation. Also the last term in the above equation is much smaller than the third term for the wind case and is equal to zero for the ISM case, so can be neglected. If we now substitute equation (32) into the above equation, we get equation (9) in $\S 2$.

The derivation of the perturbation equations of region 3 is very similar to that of region 2 . 


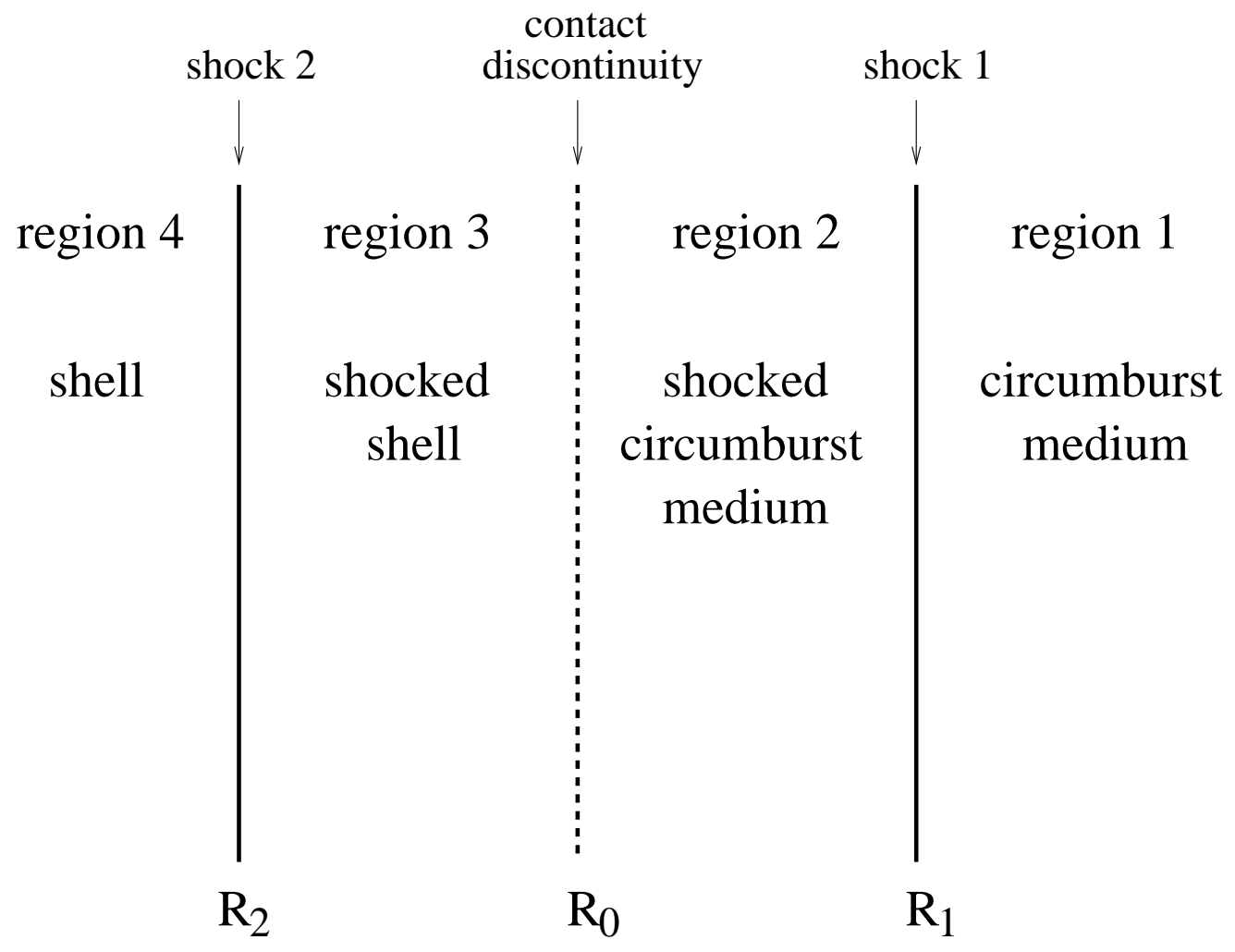

Fig. 1.- Structure of the forward/reverse shock system. 

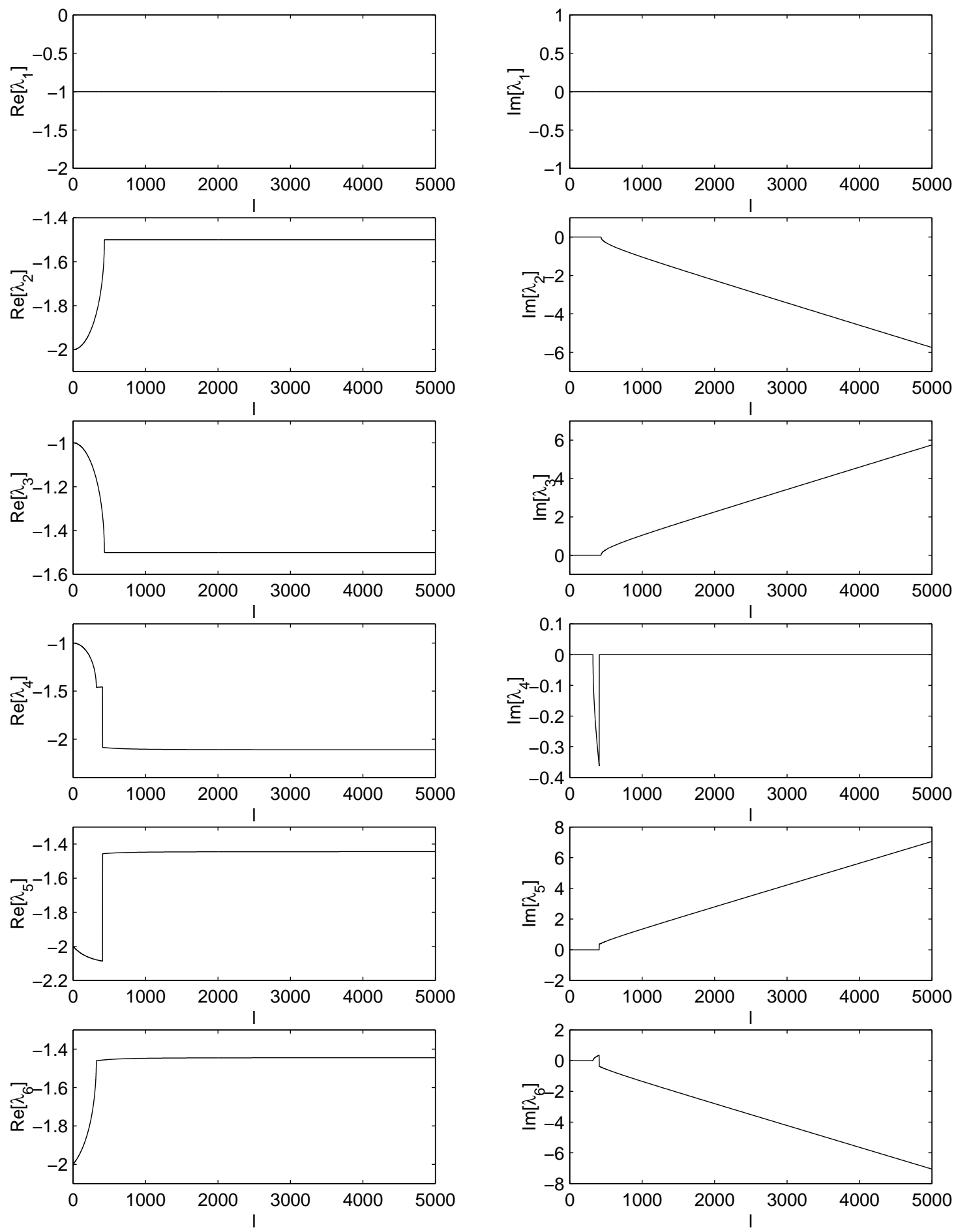

Fig. 2.- Real and imaginary parts of the six eigenvalues in the wind case as functions of $l$ with $\gamma_{c}=500$. 

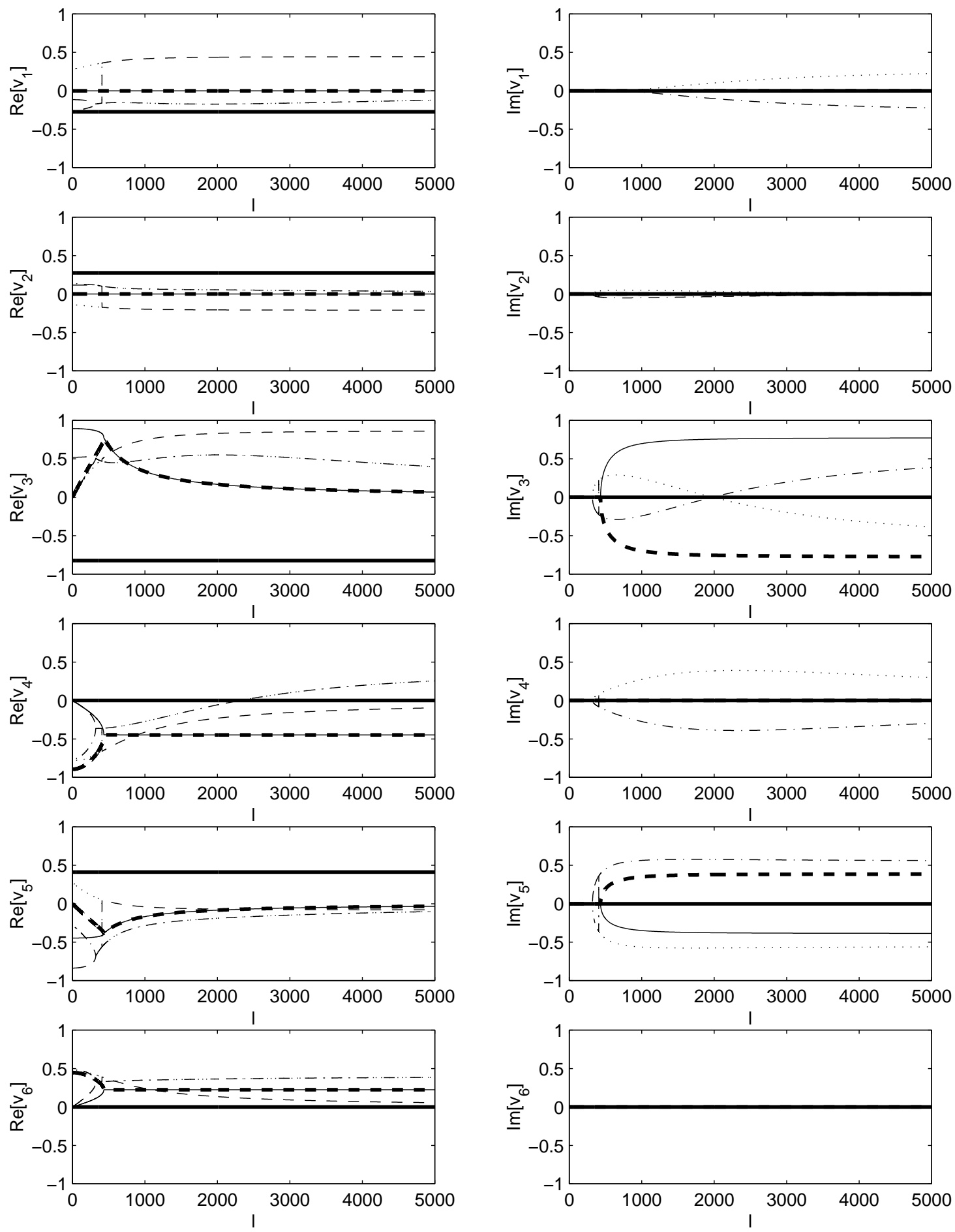

Fig. 3.- Real and imaginary parts of the six components of different eigenvectors (modes) in the wind case with $\gamma_{c}=500$. Each row corresponds to one component of the eigenvector. Different line types correspond to six different eigenvectors: the thick solid line refers to the eigenvector of $\lambda_{1}$, the thick dashed line to $\lambda_{2}$, the thin solid line to $\lambda_{3}$, the thin dashed line to $\lambda_{4}$, the dotted line to $\lambda_{5}$ and the dashed-dot line refers to $\lambda_{6}$. 

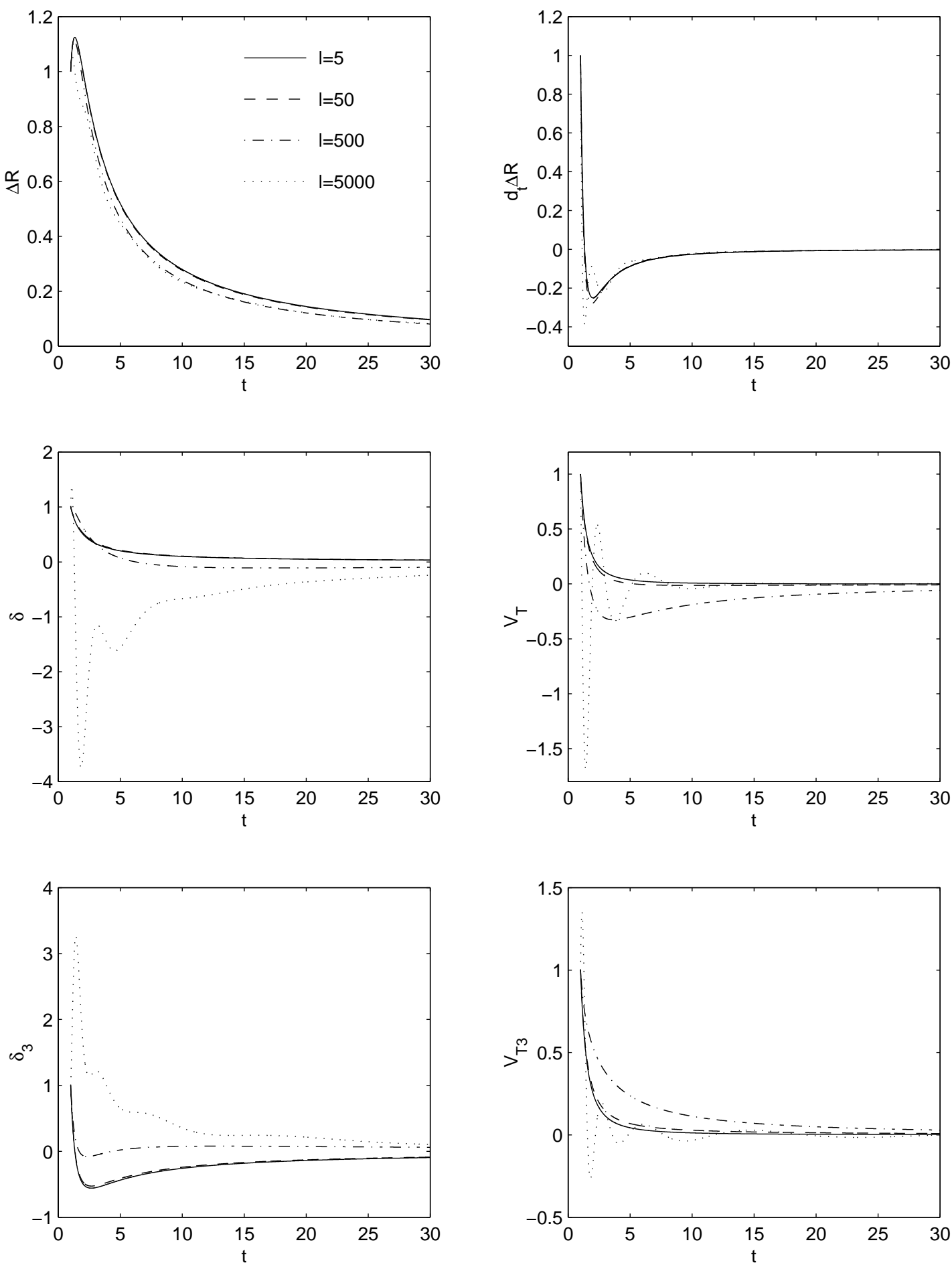

Fig. 4. - Evolution of the perturbation variables in the wind case with $\gamma_{c}=500$. Four different line types correspond to $l=5, l=50, l=500$ and $l=5 \times 10^{3}$. 

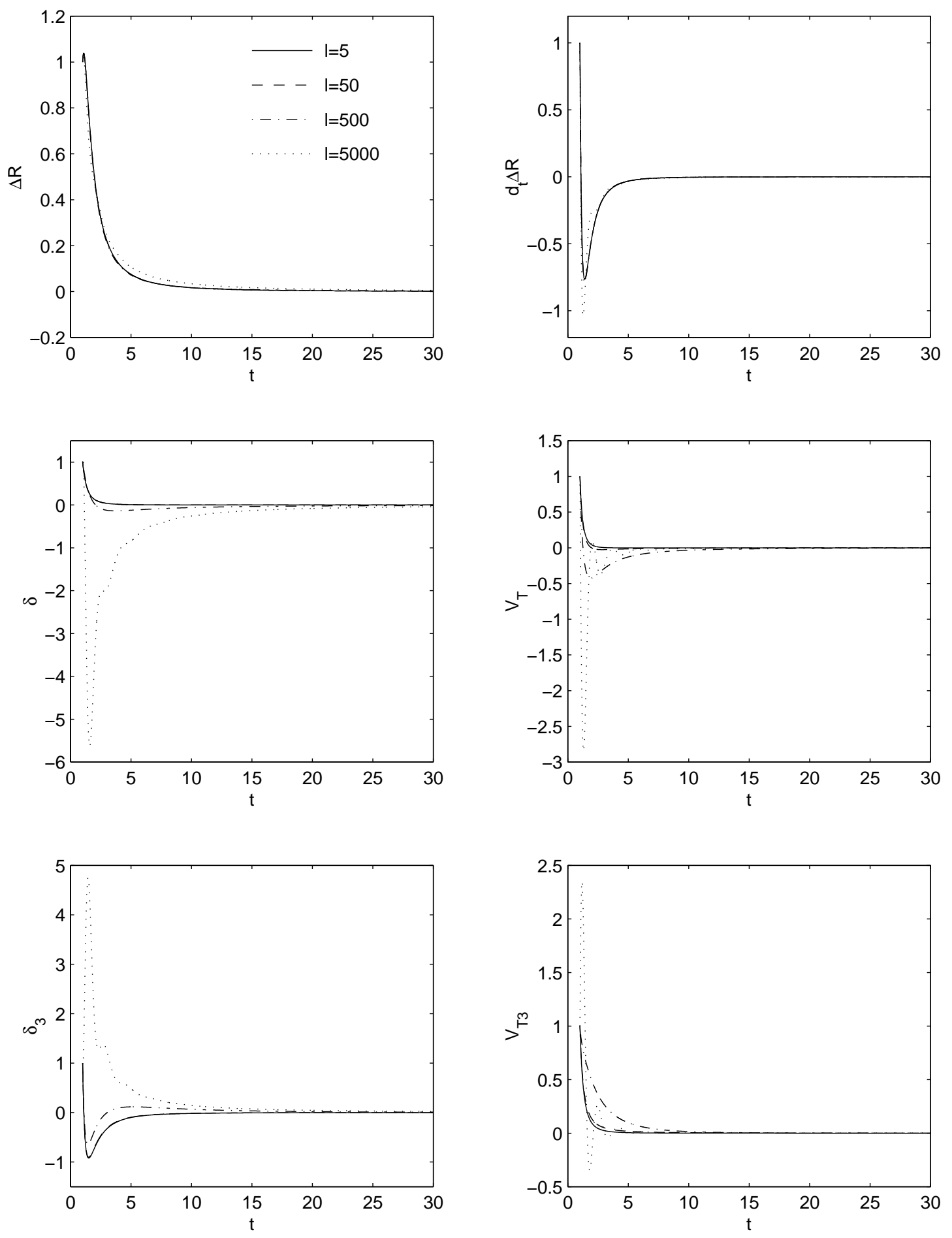

Fig. 5. - Evolution of the perturbation variables in the ISM case with $\gamma_{c}=500$. 